\title{
On the nature of faint mid-infrared sources in M 33
}

\author{
E. Corbelli ${ }^{1}$, C. Giovanardi ${ }^{1}$, F. Palla ${ }^{1}$, and S. Verley ${ }^{1,2}$ \\ 1 Osservatorio Astrofisico di Arcetri - INAF, Largo E. Fermi 5, 50125 Firenze, Italy \\ e-mail: [edvige;giova;palla]@arcetri.astro.it \\ 2 Dept. de Física Teórica y del Cosmos, Facultad de Ciencias, Universidad de Granada, Spain \\ e-mail: simon@ugr.es
}

Received 22 October 2010 / Accepted 26 January 2011

\begin{abstract}
Aims. We investigate the nature of $24 \mu \mathrm{m}$ sources in M 33 that have weak or no associated $\mathrm{H} \alpha$ emission. Both bright evolved stars and embedded star-forming regions are visible as compact infrared sources in the 8 and $24 \mu \mathrm{m}$ Spitzer maps of M 33 and contribute to the more diffuse and faint emission in these bands. Can we distinguish the two populations?

Methods. We carry out deep CO $J=2-1$ and $J=1-0$ line searches at the location of 18 compact mid-IR sources and two optically selected ones to unveil an ongoing star formation process throughout the disk of M33. In the absence of high-resolution CO maps we use different assumptions to estimate cloud masses from pointed observations. We also analyze if the spectral energy distribution and mid-IR colors can be used to discriminate between evolved stars and star-forming regions.

Results. Molecular emission is detected at the location of 17 sources at the level of $0.3 \mathrm{~K} \mathrm{~km} \mathrm{~s}^{-1}$ or higher in at least one of the $\mathrm{CO}$ rotational lines. Even though the number of giant molecular clouds drops beyond $4 \mathrm{kpc}$ in M 33, our deep observations reveal that clouds of smaller mass are common out to $6.8 \mathrm{kpc}$. Estimated cloud masses range between $10^{4}$ and $10^{5} M_{\odot}$, assuming likely values of the $\mathrm{CO}-\mathrm{to}-\mathrm{H}_{2}$ conversion factor and virial equilibrium. Sources that are known to be evolved variable stars show weaker or undetectable CO lines. Evolved stars occupy a well defined region of the IRAC color-color diagrams. Star-forming regions are scattered throughout a larger area, even though the bulk of the distribution has different IRAC colors than evolved variable stars. We estimate that about half of the $24 \mu \mathrm{m}$ sources without an $\mathrm{H} \alpha$ counterpart are genuine embedded star-forming regions. Sources with faint but compact $\mathrm{H} \alpha$ emission have an incomplete Initial Mass Function (IMF) at the high-mass end and are compatible with a population of young clusters with a stochastically sampled, universal IMF.
\end{abstract}

Key words. galaxies: individual: M 33 - local group - galaxies: star formation - ISM: clouds - ISM: molecules stars: AGB and post-AGB

\section{Introduction}

Our knowledge of molecular clouds and of the processes in the interstellar medium (hereafter ISM) that lead to the birth of stars is mostly based on Galactic studies. Local Group galaxies are however sufficiently close to allow individual massive stars and molecular clouds to be detected. M33, at a known distance, has a high star formation rate per unit area and a low overall extinction compared with M31, owing to the moderate gas content and low inclination. It is therefore an ideal laboratory for the investigation of the relationship of molecular clouds to other ISM components and evolutionary scenarios involving blue, low-luminosity galaxies. Recent high-resolution optical (HST), infrared (Spitzer; hereafter, IR) and 21-cm observations (VLA) have traced star formation and the ISM throughout the M 33 disk with high accuracy. Our investigation of the IR emission in M 33 (Verley et al. 2007, 2009, 2010; Corbelli et al. 2009) via Spitzer high-resolution images has unveiled a variety of star formation sites through infrared colors and optical-to-IR ratios. In particular, our analysis has shown the existence of two types of IR-selected sources: sources with only diffuse or very faint $\mathrm{H} \alpha$ emission, and sources with a definite $\mathrm{H} \alpha$ counterpart. In the former sample we can find sites at an early stage of massive star formation, which give us the opportunity to study individual embedded newly born HII regions in a galaxy different than our own.
Young stellar clusters before the phase of gas removal, via photoionization or stellar winds, are embedded into molecular gas and are detectable only at infrared and radio wavelengths. Previous searches in M 33 have not been successful in detecting embedded clusters. A radio-selected sample of sources in M 33 has been analyzed by Buckalew et al. (2006), who found optically visible counterparts with ages between 2-10 Myr. Similar ages have been derived by Grossi et al. (2010) by analyzing the spectral energy distribution of a sample of compact HII regions. These results point out the paucity of embedded clusters, which might be a short-lived phase of the cluster lifetime. A dust abundance lower than usual or a mass spectrum of molecular clouds steeper than in our Galaxy may be responsible for this result. Buckalew et al. (2006) suggest to analyze an IR-selected sample, an approach that is now possible thanks to M 33 Spitzer images and $24 \mu \mathrm{m}$ source catalog of Verley et al. (2007). Mid-IR sources, which have no visible counterpart in the $\mathrm{H} \alpha$ emission map, are generally faint and their nature is not obvious. Candidates include evolved clusters, evolved stars with dusty envelopes (such as pulsating asymptotic giant branch, hereafter AGBs, carbon stars, etc.), embedded star-forming sites, and small young clusters that lack massive stars and hence ionizing photons. From the available catalogs we can exclude evolved clusters (as well as planetary nebulae: see Verley et al. 2007), because these have already removed their dusty envelope. Bright evolved stars have also been cataloged, and we here discuss the 
likely presence of contamination. If there are molecular clouds around these sources, this would instead confirm ongoing star formation.

A full imaging of molecular cloud complexes in M33 has been completed by the BIMA interferometer (Engargiola et al. 2003) and the FCRAO-14m telescope (Corbelli 2003; Heyer et al. 2004) using the ${ }^{12} \mathrm{CO} J=1-0$ line. In our Galaxy, giant molecular clouds (hereafter GMCs) break up into smaller subunits when observed at high spatial resolution (Rosolowsky et al. 2003). However, in M 33 both surveys (FCRAO and BIMA) do not find any complex above the survey completeness limit $\left(\sim 10^{5} M_{\odot}\right)$ beyond a galactocentric radius of $4 \mathrm{kpc}$. On the other hand, star formation drops only at $7 \mathrm{kpc}$, well beyond the region where giant complexes are confined. Recent single-dish M 33 surveys (Gardan et al. 2007; Gratier et al. 2010) have shown that a population of low-mass molecular clouds indeed exists and becomes the dominant one beyond $4 \mathrm{kpc}$. Here, molecular clouds no longer aggregate into large complexes (there are only a few GMCs found by Gratier et al. 2011) but form predominantly in smaller mass units. Likely, this is because in this flocculent spiral spiral arms fade away around $4 \mathrm{kpc}$. This population of molecular clouds may be more easily affected or dispersed by the growth of HII regions, and therefore their properties and detectability may be strongly linked to the evolution of the associated HII region. However, because the aim of the all-disk surveys was to map large areas of the M 33 disk, their sensitivity was not sufficient to unveil the presence of molecular gas around most of the IR sources detected by Spitzer. Thus, the nature of IR sources without associated $\mathrm{H} \alpha$ emission needs additional efforts to be clarified.

For the aim of this paper we restricted our sample to a few IR sources that are isolated and are mostly located beyond $4 \mathrm{kpc}$ in the outer regions of M33. Our sample spans a variety of $F(24 \mu \mathrm{m}) / F(\mathrm{H} \alpha)$ flux ratios. The IR fluxes and sizes of the sources suggest that they may host small young clusters or $\mathrm{OB}$ associations, but also evolved stars. Although there are no GMCs associated with these sources, the IRAM-30 m telescope observations presented here gave us the opportunity to investigate whether these sources are associated to small molecular clouds (with masses $<10^{5} M_{\odot}$ ). We carried out deep searches of the ${ }^{12} \mathrm{CO} J=1-0$ and $J=2-1$ rotational lines around these sources and investigated the Spitzer IRAC colors of our sample and of a larger sample of AGBs and young clusters. There are a number of papers which investigate diagnostic IRAC colorcolor diagrams to identify various types of sources (e.g. Cohen et al. 2007; Verley et al. 2007; Gruendl \& Chu 2009). Our attempt is to distinguish evolved stars from star-forming regions in such color-color diagrams.

The plan of the paper is the following: in Sect. 2 we present the sample, the multiwavelength data set and the CO observations. We discuss in Sect. 3 the properties of detected molecular clouds and in Sect. 4 the use of IRAC color-color diagrams to distinguish between AGBs and HII regions. In Sect. 5 we use the cluster birthline to investigate the massive stellar population in our sample. The conclusions are given in Sect. 6 . 1991).

We adopt a distance to M 33 of $840 \mathrm{kpc}$ (Freedman et al.

\section{Molecular gas around selected mid-IR sources}

Dust emission can be investigated through the mid-IR and FIR data of M33 obtained with the InfraRed Array Camera (IRAC) and the Multiband Imaging Photometer (MIPS) on board Spitzer (Werner et al. 2004; Rieke et al. 2004; Fazio et al. 2004). The complete set of IRAC $(3.6,4.5,5.8$, and $8.0 \mu \mathrm{m})$ and MIPS $(24$, and $70 \mu \mathrm{m})$ images of M 33 is described in Verley et al. (2007). The image at $70 \mu \mathrm{m}$ we use is an updated version of that of Verley et al. (2007). The total number of BCD's (basic calibrated data) used in this new mosaic is 14980. The Mopex software (Makovoz \& Khan 2005) version 18.3.1 was used to combine the data. Data are from the PID 5 "M33 mapping and spectroscopy" by Gehrz (16 AORs) and the SSC (Spitzer Science Center) pipeline used to produce the BCDs is the version S16.1.0. The value of the sky background removed is 9.80 (mean value of 20 median values, each estimated in squares of $5 \times 5$ pixels, outside the galaxy). The final measured resolution is $16^{\prime \prime}$ (Gaussian $F W H M \times$ pixelsize $=13.3 \times 1.2$ ), as expected. In this new image the artificial stripes are less pronounced. To investigate the continuum ultraviolet (UV) emission of M 33, we use Galaxy Evolution Explorer (GALEX) data (Martin et al. 2005), in particular those distributed by Gil de Paz et al. (2007). A description of M33 GALEX observations in far-UV (FUV, 1350-1750 ̊) and near-UV (NUV, 1750-2750 A) and of the data reduction and calibration procedure can be found in Thilker et al. (2005).

To trace ionized gas, we adopt the narrow-line $\mathrm{H} \alpha$ image of M 33 obtained by Greenawalt (1998). The reduction process, using standard IRAF ${ }^{1}$ procedures to subtract the continuum emission, is described in detail in Hoopes \& Walterbos (2000).

\subsection{The sample}

We selected a sample of 17 IR sources from the catalog of Verley et al. (2007), whose $\mathrm{H} \alpha$ counterparts have flux intensities varying over two orders of magnitude. The $\mathrm{H} \alpha$ flux is generally modest: the brightest of these sources has a luminosity of $1.5 \times 10^{36} \mathrm{erg} \mathrm{s}^{-1}$, which corresponds to a B0 star. We cannot claim there is an $\mathrm{H} \alpha$ counterpart for the faintest $\mathrm{H} \alpha$ emission associated to a source, but only some diffuse emission. We added 3 sources located at large galactocentric radii: one visible only in the IR (s4), two selected in the H $\alpha$ map (s19, s20). In Table 1 we list the coordinates, the galactocentric distance $r$ and the emission at various wavelengths of these sources. Most of them are barely resolved in the Spitzer map at $8 \mu \mathrm{m}$ ( 2 arcsec resolution) and point-like at $24 \mu \mathrm{m}$ (6 arcsec resolution). In the $\mathrm{H} \alpha$ and FUV-GALEX maps half of them are clearly visible and have a small radius of $3-5$ arcsec. If the $A B$ magnitude in the FUV is greater than 29.5 we cannot visually identify the source in the map.

The flux in the IRAC bands, at $24 \mu \mathrm{m}$ and in $\mathrm{H} \alpha$ was measured using a varying aperture size as described in the source extraction algorithm of Verley et al. (2007). For the UV photometry we used an aperture equal to the $24 \mu \mathrm{m}$ source size. We measured the $70 \mu$ m fluxes in apertures that are 24 arcsec in diameter (equivalent to the $F W H M$ size of the IRAM-30 mt CO $J=1-0$ beam). The photometric errors are small, but owing to the occasional presence of stripes in the images we can claim detection only when the flux in the aperture is above $8.5 \mathrm{mJy}$ at $70 \mu \mathrm{m}$.

\subsection{Contamination by $A G B$ stars}

Because $24 \mu \mathrm{m}$ sources could be star-forming regions or evolved stars (see Verley et al. 2009), we now check whether some of the

1 IRAF is distributed by the National Optical Astronomy Observatories, which are operated by the Association of Universities for Research in Astronomy, Inc., under cooperative agreement with the National Science Foundation. 
Table 1. Source sample and photometry in the MIR (Spitzer), UV (GALEX), and H $\alpha$.

\begin{tabular}{|c|c|c|c|c|c|c|c|c|c|c|c|c|}
\hline ID & $\begin{array}{l}\text { RA } \\
\text { deg }\end{array}$ & $\begin{array}{l}\text { Dec } \\
\text { deg }\end{array}$ & $\begin{array}{c}r \\
\operatorname{arcmin}\end{array}$ & $\begin{array}{c}3.6 \mu \mathrm{m} \\
\mathrm{mJy}\end{array}$ & $\begin{array}{c}4.5 \mu \mathrm{m} \\
\mathrm{mJy}\end{array}$ & $\begin{array}{c}5.8 \mu \mathrm{m} \\
\mathrm{mJy}\end{array}$ & $\begin{array}{c}8.0 \mu \mathrm{m} \\
\mathrm{mJy}\end{array}$ & $\begin{array}{c}24 \mu \mathrm{m} \\
\mathrm{mJy}\end{array}$ & $\begin{array}{c}70 \mu \mathrm{m} \\
\mathrm{mJy}\end{array}$ & $\begin{array}{c}\mathrm{H} \alpha \times 10^{14} \\
\mathrm{erg} \mathrm{s}^{-1} \mathrm{~cm}^{-2}\end{array}$ & $\begin{array}{l}\text { FUV } \\
\operatorname{mag}_{A B}\end{array}$ & $\begin{array}{l}\text { NUV } \\
\operatorname{mag}_{A B}\end{array}$ \\
\hline s1 & 23.34048 & +30.60659 & 9.20 & 0.85 & 0.99 & 1.52 & 2.85 & 3.99 & $(8.2)$ & 0.23 & $30.4 \pm 3.9$ & $29.8 \pm 1.6$ \\
\hline s2 & 23.35623 & +30.59784 & 8.11 & 0.77 & 1.28 & 1.54 & 1.84 & 1.35 & $\ldots$ & 0.01 & ......... & ......... \\
\hline s3 & 23.55369 & +30.49387 & 15.17 & 1.26 & 2.40 & 3.11 & 3.56 & 3.07 & $\ldots$ & 0.16 & $30.6 \pm 4.2$ & $30.7 \pm 2.5$ \\
\hline s4 & 23.68463 & +30.28031 & 35.46 & 0.62 & 1.12 & 2.84 & 7.14 & 8.70 & 115 & & $32.4 \pm 9.6$ & $31.8 \pm 4.2$ \\
\hline s5 & 23.18128 & +30.80183 & 27.06 & 0.14 & 0.06 & 0.16 & 0.57 & 3.93 & 15.5 & 0.73 & $27.8 \pm 1.1$ & $28.2 \pm 0.8$ \\
\hline s6 & 23.39795 & +30.43258 & 14.29 & 0.54 & 0.35 & 1.81 & 5.65 & 7.55 & 30.7 & 7.27 & $27.6 \pm 1.1$ & $27.8 \pm 0.6$ \\
\hline s7 & 23.43383 & +30.80995 & 10.92 & 0.42 & 0.22 & 1.81 & 4.59 & 4.91 & 21.6 & 3.16 & $27.9 \pm 1.2$ & $28.1 \pm 0.8$ \\
\hline s8 & 23.44272 & +30.87069 & 14.59 & 0.23 & 0.18 & 0.95 & 3.18 & 6.94 & 25.5 & 5.24 & $28.7 \pm 1.7$ & $28.8 \pm 1.0$ \\
\hline s9 & 23.44566 & +30.45644 & 13.18 & 0.62 & 0.29 & 0.99 & 2.75 & 6.19 & 29.8 & 21.65 & $26.3 \pm 0.6$ & $26.4 \pm 0.3$ \\
\hline s10 & 23.49555 & +30.92542 & 16.95 & 0.19 & 0.14 & 0.71 & 2.32 & 12.55 & 18.3 & 5.44 & $28.2 \pm 1.4$ & $28.4 \pm 0.9$ \\
\hline s11 & 23.51050 & +30.96968 & 19.65 & 0.21 & 0.19 & 1.16 & 2.85 & 5.45 & 32.5 & 2.36 & $28.6 \pm 1.7$ & $28.8 \pm 1.0$ \\
\hline s12 & 23.66479 & +30.52090 & 21.20 & 0.35 & 0.16 & 2.59 & 9.37 & 21.35 & 47.7 & 1.09 & $29.0 \pm 2.0$ & $29.5 \pm 1.4$ \\
\hline s13 & 23.70374 & +30.62468 & 20.33 & 0.14 & 0.08 & 0.18 & 0.86 & 3.19 & 10.9 & 0.43 & $31.9 \pm 7.7$ & ….... \\
\hline s14 & 23.24606 & +30.69297 & 18.12 & 3.99 & 2.46 & 4.21 & 19.5 & 12.61 & 44.6 & 1.56 & $31.4 \pm 6.2$ & $30.0 \pm 1.8$ \\
\hline s15 & 23.55395 & +30.45810 & 17.34 & 0.26 & 0.19 & 0.38 & 4.85 & 14.30 & 21.1 & 0.82 & $30.6 \pm 4.3$ & $30.4 \pm 2.2$ \\
\hline s16 & 23.56527 & +30.46270 & 17.69 & 1.66 & 1.23 & 1.30 & 4.11 & 11.23 & 20.9 & 0.45 & $29.7 \pm 2.7$ & $29.5 \pm 1.4$ \\
\hline s17 & 23.61698 & +30.93344 & 18.30 & 0.20 & 0.25 & 0.52 & 1.85 & 7.33 & 12.5 & 0.28 & $30.6 \pm 4.3$ & $31.5 \pm 3.5$ \\
\hline s18 & 23.69661 & +30.63216 & 19.49 & 0.30 & 0.17 & 1.23 & 5.31 & 5.09 & 31.7 & 4.57 & $27.9 \pm 1.2$ & $28.1 \pm 0.8$ \\
\hline s19 & 23.67558 & +30.40020 & 28.08 & 0.08 & 0.06 & 0.30 & 0.95 & 0.9 & $(8.1)$ & 1.8 & $29.6 \pm 2.6$ & $29.4 \pm 1.4$ \\
\hline s20 & 23.74792 & +30.61717 & 23.99 & 0.54 & 0.71 & 0.64 & 0.95 & $\ldots$ & $\ldots$ & 4.0 & $27.8 \pm 1.2$ & $27.7 \pm 0.6$ \\
\hline
\end{tabular}

Notes. In Cols. 2-4 we also give the source right ascension, declination, and the galactocentric distance $r$.

sources in our sample have been cataloged as evolved variable stars. We cross-checked our source list with long-period variable stars cataloged by McQuinn et al. (2007) from MIR observations and with the variable point source catalog of Hartman et al. (2006) at optical wavelengths. We set the searching radius equal to the size of the $24 \mu \mathrm{m}$ emission. We found that the first three infrared-selected sources in our list (s1, s2, s3) are variables according to the classification scheme of McQuinn et al. (2007). The angular separation between the variable star and the center of the $24 \mu \mathrm{m}$ emission is less than 1.3 arcsec, i.e. 5 pc. We also found that the last source in our sample (s20, optically selected) is in the variable point-source list of Hartman et al. (2006). Given the conspicuous $\mathrm{H} \alpha$ emission associated with this region, we believe that the source s20 is an example of an evolved star close to a young cluster (at a distance of about $2 \mathrm{pc}$ ). Given its spectral characteristics, which will be shown later in the paper, s4 might also be an evolved star: a galactic AGB or an AGB in M 33 at a large galactocentric distance, beyond the area surveyed by McQuinn et al. (2007). We kept the evolved stars in our sample to check if there is any detectable $\mathrm{CO}$ emission from the surrounding region.

\subsection{The IRAM-30 mt observations}

Some CO $J=1-0$ emission has already been detected with a 45 arcsec wide beam (FCRAO) around a few sources of our sample (Heyer et al. 2004). Given the large beam size, we do not know if the detected gas is associated to or in the proximity of the sources. Therefore, we searched for $\mathrm{CO}$ emission using the smaller IRAM-30 mt beam from all sources in Table 1. The CO $J=1-0$ and $J=2-1$ lines were observed during August 2007 with a $F W H M$ beam of 24 arcsec at $115 \mathrm{GHz}$ and of 12 arc$\mathrm{sec}$ at $230 \mathrm{GHz}$. At $24 \mu \mathrm{m}$ all sources are smaller in size than the telescope beam at $230 \mathrm{GHz}$.

We observed the sources in position-switching mode, using the receiver combination A100/B100 and A230/B230 and the VESPA backend system with $240 \mathrm{MHz}$ bandwidth $(320 \mathrm{kHz}$ resolution). One source was centered on the $\mathrm{ON}$ position, another source in the OFF position. The OFF source is chosen in a region with different line-of-sight velocity than the ON position (as seen through $21-\mathrm{cm}$ maps). The spectra were smoothed to $1 \mathrm{~km} \mathrm{~s}^{-1}$ and the data from both receivers were averaged.

In Table 2 we summarize the CO data: integrated emission $I$ (in units of main-beam temperature $\mathrm{K} \mathrm{km} \mathrm{s}^{-1}$ ), mean velocity $V$, line width $\mathrm{W}$ (full width at half maximum; hereafter, $F W H M$ ) and peak intensity $\mathrm{P}$, are estimated using Gaussian fits to the lines. The rms refers to a spectral resolution of 2.2 and $1.1 \mathrm{~km} \mathrm{~s}^{-1}$ for the $\operatorname{CO} J=1-0$ and $J=2-1$, respectively. The line widths were measured using correlator spectra after correcting for hanning.

We use as the CO $J=1-0$ line intensity the average value of that derived by fitting a Gaussian to the detected emission and that obtained by summing the flux in each channel inside the signal window (determined individually for each spectrum). The result is given in Table 3. As uncertainties on the CO line intensities, we consider the highest value of the following ones: uncertainty derived from the Gaussian fit, the rms of the spectra integrated over the signal window, the dispersion between the intensity derived from Gaussian fit, and the intensity derived by the integral inside the window signal. The gain is 6.3 and 8.7 $\mathrm{Jy} \mathrm{K}^{-1}$ at 110 and $235 \mathrm{GHz}$, respectively. With these values we derive the $\mathrm{CO}$ fluxes of the two rotational levels and their ratios. In Table 3 we also quote the $\mathrm{H}_{2}$ column densities derived from FCRAO (beam $=45$ arcsec) integrated CO $J=1-0$ fluxes, using a CO- $\mathrm{H}_{2}$ conversion factor $X_{\mathrm{CO}}=2.8 \times 10^{20} \mathrm{~cm}^{-2} \mathrm{~K}^{-1}$. If there are no entries in the column corresponding to the FCRAO column density, it means that the source was outside the region mapped by FCRAO.

The bolometric luminosity for star-forming regions in Table 3 is computed as the sum of the FUV and NUV luminosities uncorrected for absorption, added to the total infrared luminosity (hereafter, TIR luminosity):

$L_{\mathrm{bol}}=L_{\mathrm{FUV}}+L_{\mathrm{NUV}}+L_{\mathrm{TIR}}+24 L_{\mathrm{H} \alpha}$,

where the $\mathrm{H} \alpha$ luminosity term accounts for the ionizing radiation (Leitherer et al. 1999) and $L_{\mathrm{TIR}}$ for the UV radiation absorbed by 
Table 2. Gaussian fits to CO lines.

\begin{tabular}{|c|c|c|c|c|c|c|c|c|c|c|}
\hline ID & $\begin{array}{c}I_{1-0} \\
\mathrm{~K} \mathrm{~km} \mathrm{~s}^{-1}\end{array}$ & $\begin{array}{c}I_{2-1} \\
\mathrm{~K} \mathrm{~km} \mathrm{~s}^{-1}\end{array}$ & $\begin{array}{c}V_{1-0} \\
\mathrm{~km} \mathrm{~s}^{-1}\end{array}$ & $\begin{array}{c}V_{2-1} \\
\mathrm{~km} \mathrm{~s}^{-1}\end{array}$ & $\begin{array}{c}W_{1-0} \\
\mathrm{~km} \mathrm{~s}^{-1}\end{array}$ & $\begin{array}{c}W_{2-1} \\
\mathrm{~km} \mathrm{~s}^{-1}\end{array}$ & $\begin{array}{c}P_{1-0} \\
\mathrm{~K}\end{array}$ & $\begin{array}{c}P_{2-1} \\
\mathrm{~K}\end{array}$ & $\begin{array}{c}\mathrm{rms}_{1-0} \\
\mathrm{~K}\end{array}$ & $\begin{array}{c}\mathrm{rms}_{2-1} \\
\mathrm{~K}\end{array}$ \\
\hline s1 & $0.284 \pm 0.028$ & $0.665 \pm 0.037$ & $-156.87 \pm 0.20$ & $-156.91 \pm 0.10$ & $2.90 \pm 0.48$ & $3.19 \pm 0.31$ & 0.063 & 0.165 & 0.008 & 0.019 \\
\hline s2 & $<0.150$ & $<0.200$ & & & & & & & 0.009 & 0.018 \\
\hline s3 & $0.247 \pm 0.025$ & $0.467 \pm 0.047$ & $-155.82 \pm 0.35$ & $-155.31 \pm 0.64$ & $5.75 \pm 1.69$ & $11.67 \pm 2.35$ & 0.039 & 0.036 & 0.008 & 0.015 \\
\hline s4 & $<0.100$ & $<0.150$ & & & & & & & 0.007 & 0.014 \\
\hline s5 & $0.665 \pm 0.038$ & $0.746 \pm 0.107$ & $-179.38 \pm 0.16$ & $-178.93 \pm 0.29$ & $4.93 \pm 0.65$ & $3.79 \pm 0.59$ & 0.109 & 0.181 & 0.011 & 0.059 \\
\hline s6 & $0.303 \pm 0.022$ & $0.639 \pm 0.038$ & $-108.04 \pm 0.10$ & $-107.89 \pm 0.08$ & $1.61 \pm 0.29$ & $1.89 \pm 0.29$ & 0.106 & 0.222 & 0.010 & 0.024 \\
\hline s7 & $1.011 \pm 0.025$ & $1.338 \pm 0.033$ & $-240.99 \pm 0.07$ & $-240.94 \pm 0.06$ & $4.99 \pm 0.27$ & $4.97 \pm 0.19$ & 0.162 & 0.244 & 0.007 & 0.015 \\
\hline s8 & $0.483 \pm 0.028$ & $0.981 \pm 0.035$ & $-243.49 \pm 0.13$ & $-243.39 \pm 0.07$ & $4.03 \pm 0.50$ & $3.33 \pm 0.21$ & 0.082 & 0.235 & 0.007 & 0.020 \\
\hline s9 & $1.206 \pm 0.028$ & $1.921 \pm 0.035$ & $-124.46 \pm 0.05$ & $-124.36 \pm 0.03$ & $3.93 \pm 0.19$ & $3.37 \pm 0.10$ & 0.235 & 0.494 & 0.009 & 0.017 \\
\hline s10 & $0.646 \pm 0.023$ & $1.762 \pm 0.034$ & $-253.66 \pm 0.08$ & $-253.87 \pm 0.04$ & $3.61 \pm 0.22$ & $3.87 \pm 0.13$ & 0.134 & 0.386 & 0.008 & 0.017 \\
\hline s11 & $1.114 \pm 0.027$ & $0.704 \pm 0.039$ & $-261.80 \pm 0.10$ & $-261.87 \pm 0.14$ & $7.64 \pm 0.65$ & $4.35 \pm 0.63$ & 0.126 & 0.128 & 0.007 & 0.017 \\
\hline s12 & $1.308 \pm 0.051$ & $1.516 \pm 0.042$ & $-151.74 \pm 0.12$ & $-151.40 \pm 0.06$ & $5.54 \pm 0.29$ & $5.30 \pm 0.30$ & 0.196 & 0.290 & 0.007 & 0.018 \\
\hline s13 & $0.809 \pm 0.024$ & $1.001 \pm 0.039$ & $-180.78 \pm 0.07$ & $-180.06 \pm 0.07$ & $3.89 \pm 0.21$ & $3.48 \pm 0.23$ & 0.163 & 0.249 & 0.008 & 0.021 \\
\hline s14 & $1.326 \pm 0.026$ & $1.191 \pm 0.035$ & $-173.56 \pm 0.05$ & $-173.50 \pm 0.06$ & $4.92 \pm 0.25$ & $3.90 \pm 0.24$ & 0.211 & 0.267 & 0.007 & 0.017 \\
\hline \multirow[t]{2}{*}{ s 15} & $0.374 \pm 0.021$ & $0.406 \pm 0.027$ & $-137.83 \pm 0.11$ & $-137.45 \pm 0.09$ & $3.11 \pm 0.66$ & $2.19 \pm 0.40$ & 0.088 & 0.133 & 0.008 & 0.017 \\
\hline & $0.150 \pm 0.020$ & $0.295 \pm 0.026$ & $-130.72 \pm 0.25$ & $-131.17 \pm 0.12$ & $2.33 \pm 1.45$ & $2.36 \pm 0.49$ & 0.039 & 0.108 & 0.008 & 0.017 \\
\hline s16 & $<0.100$ & $<0.150$ & & & & & & & 0.007 & 0.017 \\
\hline s17 & $1.10 \pm 0.029$ & $0.838 \pm 0.058$ & $-261.31 \pm 0.09$ & $-262.45 \pm 0.16$ & $6.49 \pm 0.40$ & $4.61 \pm 0.44$ & 0.141 & 0.171 & 0.008 & 0.028 \\
\hline s18 & $0.975 \pm 0.030$ & $1.259 \pm 0.036$ & $-183.14 \pm 0.08$ & $-182.97 \pm 0.06$ & $5.73 \pm 0.43$ & $4.65 \pm 0.23$ & 0.148 & 0.252 & 0.008 & 0.016 \\
\hline s19 & $0.666 \pm 0.067$ & $0.654 \pm 0.065$ & $-152.91 \pm 0.49$ & $-154.16 \pm 0.35$ & $4.80 \pm 1.08$ & $6.32 \pm 0.91$ & 0.059 & 0.093 & 0.014 & 0.028 \\
\hline s20 & $0.344 \pm 0.050$ & $0.178 \pm 0.044$ & $-187.52 \pm 0.57$ & $-190.32 \pm 0.32$ & $4.03 \pm 1.15$ & $2.03 \pm 0.86$ & 0.046 & 0.064 & 0.013 & 0.029 \\
\hline
\end{tabular}

Notes. In Cols. 1 and 2 we show the integrated intensity of the $J=1-0$ and $J=2-1$ lines respectively, in Cols. 3 and 4 the mean velocity of the two lines, in Cols. 5 and 6 the relative line widths, in Cols. 7 and 8 the peak intensities, and in the last two Cols. the rms for the CO $J=1-0$ and $J=2-1$ respectively.

Table 3. Brightness of CO clouds and associated stellar clusters.

\begin{tabular}{|c|c|c|c|c|c|c|c|c|c|c|}
\hline ID & $\begin{array}{c}\left\langle I_{1-0}\right\rangle \\
\mathrm{K} \mathrm{km} \mathrm{s}^{-1} \\
\end{array}$ & $\begin{array}{c}\left\langle I_{2-1}\right\rangle \\
\mathrm{K} \mathrm{km} \mathrm{s}^{-1} \\
\end{array}$ & $\frac{\left\langle I_{2-1}\right\rangle}{\left\langle I_{1-0}\right\rangle}$ & $\begin{array}{c}\log N_{\mathrm{H}_{2} \mathrm{FCRAO}}^{1-0} \\
\mathrm{~cm}^{-2}\end{array}$ & $\begin{array}{c}\log L_{\mathrm{H} \alpha}^{\mathrm{obs}} \\
\mathrm{erg} \mathrm{s}^{-1}\end{array}$ & $\begin{array}{c}\log L_{\mathrm{bol}}^{\mathrm{obs}} \\
\mathrm{erg} \mathrm{s}^{-1} \\
\end{array}$ & $\begin{array}{l}M_{*} \\
M_{\odot}\end{array}$ & $\begin{array}{c}\log L_{\mathrm{H} \alpha}^{\exp } \\
\operatorname{erg~s}^{-1} \\
\end{array}$ & $\begin{array}{c}A_{V} \\
\mathrm{mag}\end{array}$ & Notes \\
\hline s1 & $0.300 \pm 0.028$ & $0.658 \pm 0.037$ & 2.19 & $<20.4$ & 36.11 & $38.13^{a}$ & $\ldots$ & $\ldots$ & $\ldots$ & Visible only at $8-24-70 \mu \mathrm{m}$ \\
\hline s2 & $<0.150$ & $<0.200$ & $\ldots$ & $<20.4$ & $<33.97$ & $37.88^{a}$ & $\ldots$ & $\ldots$ & $\ldots$ & Visible only at $8-24 \mu \mathrm{m}$ \\
\hline s3 & $0.265 \pm 0.025$ & $0.306 \pm 0.228$ & 1.15 & $<20.4$ & 35.99 & $38.17^{a}$ & $\ldots$ & $\ldots$ & $\ldots$ & Visible only at $8-24 \mu \mathrm{m}$ \\
\hline s4 & $<0.100$ & $<0.150$ & $\ldots$ & $\ldots$ & 34.56 & $(39.00)^{a}$ & $(490)$ & $\left(37.1_{-1.5}^{+0.7}\right)$ & 4.2 & Visible only at $8-24-70 \mu \mathrm{m}$ \\
\hline s5 & $0.655 \pm 0.038$ & $0.718 \pm 0.107$ & 1.10 & $\ldots$ & 35.88 & 38.64 & 258 & $36.4_{-2.9}^{+1.1}$ & 0.4 & Weak $\mathrm{H} \alpha$, non isol. \\
\hline s6 & $0.290 \pm 0.022$ & $0.571 \pm 0.097$ & 1.97 & $<20.4$ & 37.11 & 39.15 & 582 & $37.2_{-1.1}^{+0.7}$ & 1.0 & \\
\hline s7 & $1.060 \pm 0.069$ & $1.363 \pm 0.035$ & 1.29 & $21.02_{-0.12}^{+0.09}$ & 36.74 & 38.98 & 431 & $37.0_{-2.0}^{+0.8}$ & 0.9 & \\
\hline s8 & $0.565 \pm 0.115$ & $0.947 \pm 0.047$ & 1.68 & $21.18_{-0.14}^{+0.10}$ & 37.13 & 39.00 & 490 & $37.1_{-1.5}^{+0.7}$ & 1.4 & \\
\hline s9 & $1.229 \pm 0.033$ & $1.861 \pm 0.085$ & 1.51 & $<20.4$ & 37.32 & 39.30 & 794 & $37.4_{-0.8}^{+0.6}$ & 0.3 & \\
\hline s10 & $0.642 \pm 0.023$ & $1.765 \pm 0.034$ & 2.75 & $20.90_{-0.15}^{+0.11}$ & 37.07 & 39.04 & 504 & $37.1_{-1.5}^{+0.7}$ & 1.2 & \\
\hline s11 & $1.087 \pm 0.038$ & $0.659 \pm 0.064$ & 0.61 & $\ldots$ & 36.73 & 38.86 & 370 & $36.9_{-2.4}^{+0.8}$ & 1.2 & \\
\hline s12 & $1.282 \pm 0.051$ & $1.681 \pm 0.283$ & 1.31 & $20.65_{-0.26}^{+0.16}$ & 36.83 & 39.30 & 794 & $37.4_{-0.8}^{+0.6}$ & 2.3 & No FUV, tail \\
\hline s13 & $0.888 \pm 0.112$ & $1.000 \pm 0.039$ & 1.13 & $21.23_{-0.07}^{+0.05}$ & 36.58 & 38.47 & 215 & $36.0_{-2.8}^{+1.3}$ & 2.7 & No FUV \\
\hline s14 & $1.422 \pm 0.135$ & $1.263 \pm 0.102$ & 0.89 & $20.78_{-0.22}^{+0.15}$ & 37.69 & 39.49 & 948 & $37.6_{-0.7}^{+0.4}$ & 4.1 & No FUV \\
\hline s15 & $0.372 \pm 0.020$ & $0.352 \pm 0.016$ & 0.95 & $<20.4$ & 36.99 & 39.11 & 566 & $37.2_{-1.2}^{+0.7}$ & 3.0 & No FUV, weak $\mathrm{H} \alpha$ \\
\hline s15 & $0.159 \pm 0.020$ & $0.315 \pm 0.028$ & 1.98 & $<20.4$ & 36.99 & 39.11 & 566 & $37.2_{-1.2}^{+0.7}$ & 3.0 & No FUV, weak $\mathrm{H} \alpha$ \\
\hline s16 & $<0.100$ & $<0.150$ & $\ldots$ & $<20.4$ & 36.41 & 38.98 & 431 & $37.0_{-2.0}^{+0.8}$ & 2.3 & Weak $\mathrm{H} \alpha$ \\
\hline s17 & $1.159 \pm 0.082$ & $0.852 \pm 0.058$ & 0.74 & $21.13_{-0.15}^{+0.11}$ & 36.29 & 38.72 & 313 & $36.5_{-2.7}^{+1.1}$ & 2.5 & Visible only at $8-24-70 \mu \mathrm{m}$ \\
\hline s18 & $1.012 \pm 0.052$ & $1.269 \pm 0.036$ & 1.25 & $20.60_{-0.39}^{+0.21}$ & 36.93 & 39.03 & 500 & $37.1_{-1.5}^{+0.7}$ & 1.0 & \\
\hline s19 & $0.651 \pm 0.067$ & $0.637 \pm 0.065$ & 0.98 & $\ldots$ & 36.48 & 38.38 & 206 & $35.8_{-2.7}^{+1.5}$ & 0.9 & IR weak \\
\hline s20 & $0.353 \pm 0.050$ & $0.172 \pm 0.044$ & 0.49 & $\ldots$ & 36.48 & 38.54 & 241 & $36.3_{-2.8}^{+1.2}$ & 0.04 & IR weak \\
\hline
\end{tabular}

Notes. In Cols. 2 and 3 we give the value of the CO $J=1-0$ and 2-1 line intensity averaged between that derived from Gaussian fits and that obtained by summing the flux in each channel. The ratio between the two CO line intensities is shown in Col. 4. In Col. 5 we give the $\mathrm{H}_{2}$ column densities derived from FCRAO observations and in Cols. 6 and 7 the $\mathrm{H} \alpha$ luminosity (corrected for extinction) and the bolometric luminosity of the sources. In the last three Cols. we show the estimated mass, expected $\mathrm{H} \alpha$ luminosity (see text for details), and visual extinction of the stellar clusters. ${ }^{(a)}$ Luminosity as from Eq. (3), because the source is an AGB star. ${ }^{(b)}$ Source of unknown nature, luminosity computed according to Eq. (1). ${ }^{(c)}$ HII region next to an AGB star, luminosity computed according to Eq. (1). 
grains and re-emitted in the IR. We did not considered the continuum radiation longward of $2800 \AA$ which becomes important when young clusters have luminosities lower than $10^{38} \mathrm{erg} \mathrm{s}^{-1}$. The estimated TIR luminosity, $L_{\mathrm{TIR}}$, was computed following Verley et al. (2009):

$\log L_{\mathrm{TIR}}=\log L_{24}+1.08+0.51 \times \log \frac{L_{v, 8}}{L_{v, 24}}$,

and the luminosity function from the 8 and $24 \mu \mathrm{m}$ fluxes as $v L_{v}$. The above expression is correct for HII regions whose IR emission peaks longward of $24 \mu \mathrm{m}$. Evolved stars s1, s2, s3 have no emisson in the FIR and the above formula overestimates the TIR. For these sources we computed the luminosity according to Groenewegen et al. (2007) who have shown that the dust emission from evolved stars closely follows that of a black body at temperatures of 400-600 K. In particular, using the 8 and $24 \mu \mathrm{m}$ fluxes given in Table 1, we estimate the luminosity in solar luminosity units as

$L_{\text {bol }}(\mathrm{AGB})=130 \frac{F_{8}}{\mathrm{mJy}}\left(\frac{F_{8}}{F_{24}}\right)^{1.76} \exp \left(4.4\left(F_{24} / F_{8}\right)^{0.44}\right)$.

For s4 we give the luminosities and stellar masses using the formulae for HII regions, but put the values in parenthesis because it may be an evolved star. Extinction corrections for $\mathrm{H} \alpha$ fluxes in HII regions are $0.83 \mathrm{~A}_{V}$ where $\mathrm{A}_{V}$, the visual extinction, is given as

$A_{V}=3 \times 0.57 \times \log \left(\frac{L_{\mathrm{TIR}}}{L_{\mathrm{NUV}}+L_{\mathrm{FUV}}}+1\right)$.

In Table 3 we list extinction values and cluster masses. Following Corbelli et al. (2009), we set $0.1 M_{\odot}$ as the lower limit of the IMF and use a Salpeter slope of 2.3 down to $0.5 M_{\odot}$, and 1.3 between 0.5 and $0.1 M_{\odot}$. When the bolometric luminosities are low, as in the case of these clusters, the upper end of the IMF is not fully populated and one has to take into account stochastic effects. In the stochastic regime, uncertainties are large and a simple scaling law between luminosity and cluster mass does not apply (and it would underestimate the cluster mass). For $L_{\text {bol }}<10^{40} \mathrm{erg} \mathrm{s}^{-1}$ the possible range of cluster masses for a given $L_{\mathrm{bol}}$ is up to one order of magnitude (Corbelli et al. 2010). In Table 3 we show the median cluster mass for the given bolometric luminosity, the expected median $\mathrm{H} \alpha$ luminosity, $L_{\mathrm{H} \alpha}^{\exp }$ (corresponding to the observed $L_{\mathrm{bol}}$ ) and the relative uncertainties. The observed values corrected for extinction, $L_{\mathrm{H} \alpha}^{\mathrm{obs}}$, are also given. Finally, we comment when there is no clear peak in the images at the location of the $24 \mu \mathrm{m}$ source.

In the next section and in the rest of this section we discuss possible correlations between cloud properties. We quote in parenthesis the Pearson linear correlation coefficient $r_{\mathrm{P}}$ for data with respect to star-forming regions, excluding AGBs.

The ratio of line intensities $I_{2-1} / I_{1-0}$ in Fig. 1 correlates with the ratio of line-widths $\left(r_{\mathrm{P}}=0.74\right.$ for $\log W_{2-1} / W_{1-0}-$ $\left.\log I_{2-1} / I_{1-0}\right)$. If we exclude $\mathrm{s} 19$, the source with the largest $W_{2-1} / W_{1-0}$, the Pearson linear correlation coefficient is 0.91 and the slope is $1.9 \pm 0.2$. The observed line ratio shows a marginal decrease as the galactocentric radius increases $\left(r_{\mathrm{P}}=-0.46\right)$ and it does not correlate with the cluster bolometric luminosity. In Fig. 1 we can also see that the emission at $70 \mu \mathrm{m}$ increases with the cluster mass, as expected, since for most of our clusters the infrared luminosity drives the value of the bolometric luminosity (even though we have not used the $70 \mu \mathrm{m}$ emission to compute

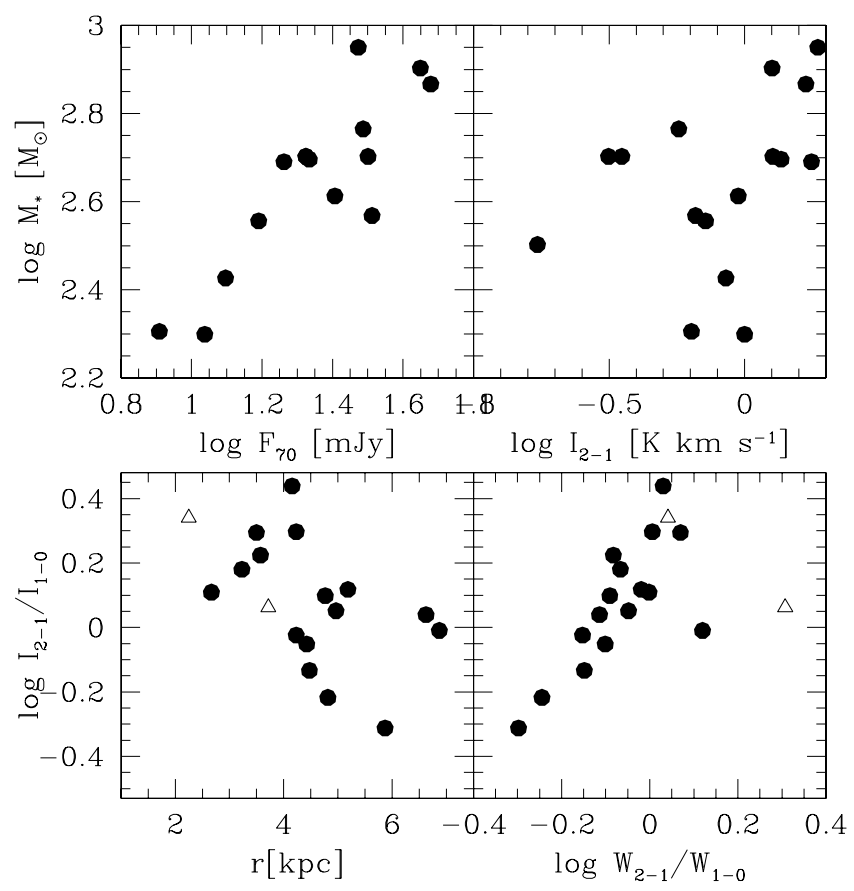

Fig. 1. Correlation of $F_{70}$ and the cluster mass is shown in the upper left panel. The upper right panel shows that clusters of larger mass are associated with brighter $\mathrm{CO} J=2-1$ lines. The bottom panels display the marginal correlation between the line intensity ratio $I_{2-1} / I_{1-0}$ and the galactocentric radius and the good correlation between $I_{2-1} / I_{1-0}$ and the line width ratio $W_{2-1} / W_{1-0}$. Triangles are for variable stars $(\mathrm{s} 1, \mathrm{~s} 3)$ and are displayed only in the lower panels.

the TIR). The scatter is large however $\left(r_{\mathrm{P}}=0.46\right)$. The brightness of the $\mathrm{CO}$ lines does not correlate with the median cluster mass $\left(r_{\mathrm{P}}=0.38\right)$, even though Fig. 1 shows that more massive clusters are associated with the brightest $\mathrm{CO} J=2-1$ lines.

\section{Three methods for computing molecular masses and sizes}

We computed the properties of the molecular gas following three different methods. In practice, we have three unknowns: the cloud size, the conversion factor $X_{\mathrm{CO}}$ between the CO luminosity and the $\mathrm{H}_{2}$ surface density, and $R$ the intrinsic line ratio. The latter quantity is the ratio between the $J=2-1$ and $J=1-0$ lines we would measure by mapping the cloud with a telescope beam smaller than the cloud size. The expected line ratio is the line ratio we expect to observe knowing the telescope beam size at the two line frequencies, the intrinsic line ratio and the source extent. The observed line ratio is the measured value of $I_{2-1} / I_{1-0}$. If we consider the cloud virialized and centered at the source location, we have two equations for these three variables: one equation is given by equating the observed to the expected line ratio and the other one by equating the virial mass to the mass inferred from the $\mathrm{CO}$ line brightness. Clearly, even in this case some assumption is needed to derive the cloud properties such as mass, density, size etc. We now describe three different methods to derive cloud parameters and the underlying assumptions.

\subsection{Uniform intrinsic line ratio}

We assume a fixed intrinsic line ratio $R$ between the CO $J=2-1$ and 1-0 lines for all sources and compute the expected line ratio under the hypothesis that the source is isolated and centered 
in the beam. We infer the source diameter $D$ by equating the expected ratio to the observed one:

$R=\frac{I_{2-1}\left(1-\exp \left(-D^{2} /\left(1.2 \Theta_{1-0}\right)^{2}\right)\right)}{I_{1-0}\left(1-\exp \left(-D^{2} /\left(1.2 \Theta_{2-1}\right)^{2}\right)\right)}$

where $\Theta$ is the half-width half-maximum of the beam. Knowing the size we can infer the virial mass and by equating the virial mass to the mass derived using the $\mathrm{CO}$ line luminosity (which is the same for the 2-1 and 1-0 line once we correct for beam dilution), we infer $X_{\mathrm{CO}}$. Other cloud properties, such as the $\mathrm{H}_{2}$ volume or column density, then follow. The cloud virial mass, for a density profile which varies inversely to the cloud radius, can be written as (Wilson \& Scoville 1990)

$\frac{M_{\mathrm{vir}}}{M_{\odot}}=99 \frac{D}{\mathrm{pc}}\left(\frac{W}{\mathrm{~km} \mathrm{~s}^{-1}}\right)^{2}=406 \frac{D}{\operatorname{arcsec}}\left(\frac{W}{\mathrm{~km} \mathrm{~s}^{-1}}\right)^{2}$,

where $W$ is the $F W H M$ of the CO line and D is the diameter or cloud size. The average volume density is

$\frac{n_{\mathrm{H}_{2}}}{\mathrm{~cm}^{-3}}=\frac{406 D_{\mathrm{arcsec}} W_{\mathrm{km} \mathrm{s}^{-1}}^{2} 2 \times 10^{33}}{D_{\operatorname{arcsec}}^{3} 1.05 \times 10^{57} 1.33 \times m_{\mathrm{H}_{2}}}=174 \frac{W_{\mathrm{km} \mathrm{s}^{-1}}^{2}}{D_{\mathrm{arcsec}}^{2}}$,

where the factor 1.33 accounts for helium. The average intrinsic line ratio in molecular clouds of our Galaxy varies between 0.5 and 0.8 (Oka et al. 1998), being higher at low galactic latitudes. In external galaxies intrinsic ratios are as low as 0.3 or as high as 2 (e.g. Sorai et al. 2001; Crosthwaite \& Turner 2007) and can be explained in terms of variations of the cloud physical conditions (Sakamoto et al. 1994). Low values are expected when the gas has a low density or a low kinetic temperature. In Table 4 we give the cloud parameters derived with the first method using a uniform intrinsic line ratio $R$ equal to 0.5 . We do not give the cloud size when the line ratio is higher than the maximum possible value (observable for a point source at the beam center) or lower than the intrinsic line ratio (observable for an extended source). For $R=0.5$ estimated sizes and masses come out large enough that $\mathrm{CO}$ all disk surveys of M33 should have detected most of them. Volume densities are generally lower than $10^{3} \mathrm{~cm}^{-3}$. Because we are searching for molecular gas around sources, one may wonder whether the assumed value of the intrinsic line ratio is reasonable. The intrinsic intensity ratio can indeed take values significantly higher than unity when the emitting regions are warm $(T>40 \mathrm{~K})$, dense $\left(n_{\mathrm{H}_{2}}>10^{3} \mathrm{~cm}^{-3}\right)$ and moderately opaque $(\tau<5)$. However, higher values of $R$ would imply even more massive clouds and lower densities. Thus we conclude that it is unlikely that the intrinsic ratio $R$ is much higher than 0.5 except for the two clouds whose observed line ratio is higher than 2. Similarly, for s20, the intrinsic ratio is expected to be lower than 0.5 .

Molecular masses from to the integrated $\mathrm{CO}$ line brightness are computed according to:

$\frac{M_{\mathrm{CO}}}{M_{\odot}}=41 \frac{X_{\mathrm{CO}}}{2 \times 10^{20}} D_{\operatorname{arcsec}}^{2} \frac{I}{1-\exp \left(-D_{\operatorname{arcsec}}^{2} /(1.2 \Theta)^{2}\right.}$,

where the line intensity $I$ refers to either the CO $J=1-0$ or $J=2-1$ line. Cloud masses derived for a constant conversion factor $X_{\mathrm{CO}}=2.8 \times 10^{20} \mathrm{~cm}^{-2} \mathrm{~K}^{-1}$ are generally smaller than virial masses (except for one source in s15). Our estimated cluster masses (see Table 4) are too small to account for the difference, even taking into account the incompleteness of the IMF. In the last column we give $X_{\mathrm{CO}}$, normalized to $10^{20} \mathrm{~cm}^{-2} \mathrm{~K}^{-1}$, as the value needed to have the $\mathrm{CO}$-luminosity mass equal to virial
Table 4. Cloud parameters for an intrinsic line ratio $R=0.5$.

\begin{tabular}{ccccccc}
\hline \hline ID & $\begin{array}{c}\mathrm{D} \\
\mathrm{pc}\end{array}$ & $\begin{array}{c}M_{\mathrm{vir}}^{1-0} \\
10^{4} M_{\odot}\end{array}$ & $\begin{array}{c}M_{\mathrm{vir}}^{2-1} \\
10^{4} M_{\odot}\end{array}$ & $\begin{array}{c}n_{\mathrm{H}_{2}}^{1-0} \\
\mathrm{~cm}^{-3}\end{array}$ & $\begin{array}{c}N_{\mathrm{H}_{2}} \\
10^{20}\end{array}$ & $\begin{array}{c}X_{\mathrm{CO}} \\
10^{20} \mathrm{~cm}^{-2} \mathrm{~K}^{-1}\end{array}$ \\
\hline s1 &.. &.. &.. &.. &.. &.. \\
s2 &.. &.. &.. &.. &.. &.. \\
s3 & 77 & 26. & 107. & 15 & 36 & 58 \\
s4 &.. &.. &.. &.. &.. &.. \\
s5 & 81 & 20. & 12. & 10 & 25 & 5.6 \\
s6 & 12 & 0.31 & 0.42 & 54 & 20 & 0.7 \\
s7 & 69 & 17. & 17. & 15 & 32 & 4.0 \\
s8 & 41 & 6.7 & 4.5 & 28 & 35 & 2.4 \\
s9 & 53 & 8.2 & 6.0 & 16 & 26 & 1.6 \\
s10 &.. &.. &.. &.. &.. &.. \\
s11 & 155 & 89. & 29. & 7 & 33 & 7.3 \\
s12 & 57 & 18. & 13. & 27 & 47 & 3.2 \\
s13 & 81 & 12. & 9.7 & 7 & 18 & 2.9 \\
s14 & 102 & 25. & 15.6 & 7 & 22 & 3.0 \\
S15 & 98 & 9.3 & 4.6 & 4 & 12 & 4.0 \\
s15 & 8 & 0.52 & 0.54 & 168 & 41 & 1.0 \\
s16 &.. &.. &.. &.. &.. &.. \\
s17 & 114 & 48. & 24. & 9 & 32 & 6.0 \\
s18 & 73 & 23. & 15.4 & 19 & 43 & 2.8 \\
s19 & 94 & 22. & 37. & 8 & 23 & 9.6 \\
s20 &.. &.. &.. &.. &.. &.. \\
\hline
\end{tabular}

Notes. The cloud diameter is given in Col. 2 and the virial masses corresponding to the $J=1-0$ and $J=2-1$ CO lines are given in Cols. 3 and 4. The $\mathrm{H}_{2}$ volume and column density are shown in Cols. 5 and 6 respectively and the $\mathrm{CO}-$ to- $\mathrm{H}_{2}$ conversion factor in the last column (average value of those with respect to the two $\mathrm{CO}$ lines).

mass (we give the average of the values with respect to the two CO lines):

$X_{\mathrm{CO}}=\frac{M_{\mathrm{vir}}}{M_{\mathrm{CO}}}=10 \frac{W^{2}}{D} \frac{1-\exp \left(-D_{\mathrm{arcsec}}^{2} /\left(1.2 \Theta_{\mathrm{arcsec}}\right)^{2}\right)}{I}$,

where the values of $I, \Theta, W$ refer to the CO line that was used. The large cloud sizes derived with this method for $R=0.5$ imply large cloud masses and very low star formation efficiencies. Gas densities are lower than expected for typical molecular cloud conditions (see Sect. 3.4 for a comparison with Milky Way clouds).

We do not find a correlation between the cluster mass and the virial mass or between the ratio $M_{\mathrm{vir}}^{2-1} / M_{\mathrm{vir}}^{1-0}$ and the galactocentric radius $\left(r_{\mathrm{P}}=-0.15\right)$. For this model the $\mathrm{CO}-\mathrm{to}-\mathrm{H}_{2}$ conversion factor, $X_{\mathrm{CO}}$, does not correlate with the galactocentric radius $\left(r_{\mathrm{P}}=0.34\right)$, but there is a correlation between $X_{\mathrm{CO}}$ and the cloud mass $\left(r_{\mathrm{P}}=0.92\right)$, shown in Fig. 2. This last correlation implies that $X_{\mathrm{CO}}$ increases for more massive clouds. Can this be justified? The $X_{\mathrm{CO}}$ value is expected to increase as the extinction through the cloud or the mean volume density decreases (Maloney \& Black 1988; Glover \& Mac Low 2011). Gas densities for this model decrease as clouds get more massive, which justifies the observed trend. Finally we find a marginal correlation between the cloud size and the CO linewidth, $D \propto W^{2}$, as it is observed in resolved molecular clouds and discussed in Sect. $3.4\left(r_{\mathrm{P}}=0.61\right.$, slope $1.9 \pm 0.6$ for the $J=1-0$ line and $r_{\mathrm{P}}=0.57$ slope $1.9 \pm 0.7$ for the $J=2-1$ line).

This method heavily depends on the assumption that clouds are located at the beam center, which coincides with the peak of the IR-24 $\mu \mathrm{m}$ emission. Even though the molecular gas will likely follow the dust distribution, the peak of the mid-IR emission depends on the intensity of the radiation field that heats the grains. Consequently the molecular cloud may be offset with respect to the IR emission peak. 
E. Corbelli et al.: On the nature of faint mid-infrared sources in M33

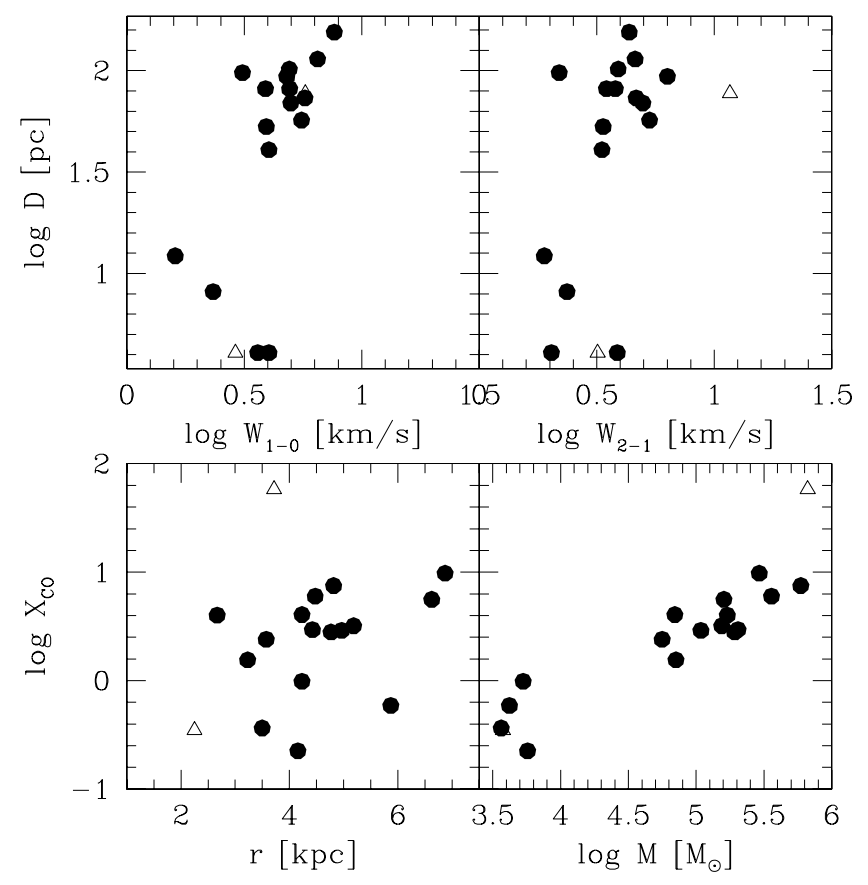

Fig. 2. Correlations using the parameters derived with the first method: in the upper panels we plot the line-width of the $J=1-0$ and 2-1 line versus the cloud size. The $X_{\mathrm{CO}}$ factor is plotted as a function of galactocentric radius and of cloud mass in lower left and right panels respectively.

\subsection{Hydrostatic equilibrium}

If the cloud turbulent pressure equals the ISM ambient pressure given by hydrostatic equilibrium, we can estimate the gas volume density of the cloud. This is in reality a lower limit because cloud cores can be denser and self-gravitating, with the outer parts close to equilibrium with the surrounding ISM. From the minimum gas density we infer $D^{\max }$, the maximum cloud size, using the virial equation. As we discuss below in Sect. 3.4, it could be that only part of the cloud is gravitationally bound and in virial equilibrium. In this case the virial equation applies only for the higher density core with size $D<D^{\max }$.

In Table 5 we give the resulting cloud parameters and below some details of this model. We compute the ambient pressure as

$P_{\mathrm{ISM}}=\frac{\pi}{2} G \Sigma_{\mathrm{g}}\left(\Sigma_{\mathrm{g}}+\frac{c_{\mathrm{g}}}{c_{\mathrm{S}}} \Sigma_{\mathrm{s}}\right) \mathrm{cm}^{-3} \mathrm{~K}$

(Corbelli 2003), where $\Sigma_{\mathrm{g}, \mathrm{s}}$ are the gas and stellar surface mass densities and $c_{\mathrm{g}, \mathrm{s}}$ are the velocity dispersion with respect to gas and stellar disk. The velocity dispersion of the gaseous disk is $c_{\mathrm{g}}=8 \mathrm{~km} \mathrm{~s}^{-1}$. We use the local values of the HI column densities for the gas surface densities (WSRT data) and a scale height of the stellar disk $z_{0}=0.5 \mathrm{kpc}$ for the stellar dispersion. The stellar dispersion and surface density derived through the dynamical analysis of the rotation curve (Corbelli 2003) reads

$c_{\mathrm{S}}=\left(\pi G z_{0} \Sigma_{\mathrm{s}}\right)^{0.5} \quad \Sigma_{\mathrm{s}}=430 \exp (-R(\mathrm{kpc}) / 1.42) M_{\odot} / \mathrm{pc}^{2}$.

The ISM pressure in M 33 does not vary much radially because the gas surface density has a very large scale length. However, it can vary locally because of filaments and flocculent spiral arms. For each source the turbulent pressure is

$P_{\text {tur }}=1.16 n_{\mathrm{H}_{2}} T_{\text {Turb }}=25 n_{\mathrm{H}_{2}} W^{2}\left(\mathrm{~km} \mathrm{~s}^{-1}\right) \mathrm{cm}^{-3} \mathrm{~K}$
Table 5. Clouds in pressure equilibrium with the surrounding ISM.

\begin{tabular}{cccccccc}
\hline \hline ID & line & $R^{\max }$ & $\begin{array}{c}D^{\max } \\
\mathrm{pc}\end{array}$ & $\begin{array}{c}M^{\max } \\
10^{4} M_{\odot}\end{array}$ & $\begin{array}{c}n_{\mathrm{H}_{2}}^{\min } \\
\mathrm{cm}^{-3}\end{array}$ & $\begin{array}{c}N_{\mathrm{H}_{2}}^{\min } \\
10^{20}\end{array}$ & $\begin{array}{c}X_{\mathrm{CO}}^{\max } \\
10^{20}\end{array}$ \\
\hline s1 & $1-0$ & 0.66 & 41 & 3.5 & 14. & 18 & 6.4 \\
s2 & $\ldots$ & & & & & & \\
s3 & $1-0$ & 0.66 & 106 & 35. & 8.6 & 28 & 53. \\
s4 & $\ldots$ & & & & & & \\
s5 & $2-1$ & 0.57 & 94 & 14. & 4.6 & 26 & 8.9 \\
s6 & $1-0$ & 0.50 & 10 & 0.2 & 80 & 24 & 0.5 \\
s7 & $2-1$ & 0.58 & 81 & 20. & 11 & 28 & 8.9 \\
s8 & $2-1$ & 0.50 & 41 & 4.7 & 18 & 23 & 4.6 \\
s9 & $2-1$ & 0.43 & 36 & 4.1 & 26 & 29 & 1.9 \\
s10 & $1-0$ & 0.88 & 49 & 6.4 & 15 & 23 & 5.4 \\
s11 & $2-1$ & 0.22 & 61 & 5.9 & 15 & 28 & 5.5 \\
s12 & $2-1$ & 0.63 & 90 & 25. & 11 & 30 & 8.6 \\
s13 & $2-1$ & 0.39 & 57 & 6.9 & 11 & 19 & 4.0 \\
s14 & $2-1$ & 0.27 & 41 & 6.3 & 25 & 31 & 2.5 \\
s15 & $2-1$ & 0.24 & 13 & 0.6 & 85 & 33 & 1.0 \\
s15 & $1-0$ & 0.51 & 14 & 0.8 & 76 & 33 & 2.9 \\
s16 & $\ldots$ & & & & & & \\
s17 & $2-1$ & 0.31 & 77 & 16. & 11 & 26 & 6.7 \\
s18 & $2-1$ & 0.47 & 65 & 14. & 15 & 30 & 7.0 \\
s19 & $1-0$ & 0.77 & 147 & 34. & 3.1 & 14 & 15. \\
s20 & $2-1$ & 0.13 & 23 & 0.9 & 23. & 16 & 1.5 \\
\hline
\end{tabular}

Notes. The CO rotational line used to infer the cloud parameters is given in Col. 2, the maximum value of the intrinsic $\mathrm{CO}$ line ratio in Col. 3 and the maximum allowed cloud diameter in Col. 4. The maximum cloud mass and the corresponding minimum volume and column density of $\mathrm{H}_{2}$ are given in Cols. 5-7 respectively. The maximum value of the COto- $\mathrm{H}_{2}$ conversion factor is shown in the last Col.

(1.16 accounts for Helium, see also Spitzer 1978, p. 37). The gas volume density is then

$n_{\mathrm{H}_{2}}^{\min }=\frac{P_{\mathrm{ISM}}\left(\mathrm{cm}^{-3} \mathrm{~K}\right)}{25 W^{2}\left(\mathrm{~km} \mathrm{~s}^{-1}\right)}$

We use the smallest linewidth measured in the $1-0$ or $2-1$ line, which gives the highest volume density. We compute the maximum cloud size by equating the virial mass to the mass in pressure equilibrium, which reads

$D_{\operatorname{arcsec}}^{\max }=\sqrt{\frac{174 W^{2}\left(\mathrm{~km} \mathrm{~s}^{-1}\right)}{n_{\mathrm{H}_{2}}^{\min }}}=66 \frac{W^{2}\left(\mathrm{~km} \mathrm{~s}^{-1}\right)}{\sqrt{P_{\mathrm{ISM}}}}$.

This relation naturally explains the size-linewidth relation $W \propto$ $\sqrt{D}$. Indeed, we find that the $2-1$ linewidth (which is mostly used in this relation) correlates with the source size, with the right slope (see Fig. 3, $r_{\mathrm{P}}=0.94$ slope $=2.0 \pm 0.2$ ). A similar relation exists for the $1-0$, but with a larger scatter $\left(r_{\mathrm{P}}=0.81\right)$. The size-linewidth relation implies another important feature often mentioned about molecular clouds, namely their fairly constant column densities. For this model the gas column density reads

$\frac{N_{\mathrm{H} 2}^{\min }\left(\mathrm{cm}^{-2}\right)}{10^{20}}=0.126 D_{\operatorname{arcsec}}^{\max } n_{\mathrm{H}_{2}}^{\min }\left(\mathrm{cm}^{-3}\right) \propto \sqrt{P_{\mathrm{ISM}}}$.

Values of the local ISM pressure in M33 do not vary much, which implies almost constant column densities, as can be seen in Table 5. We can also infer the maximum value of $X_{\mathrm{CO}}$ from the virial mass to the CO-luminosity mass ratio using the maximum size D:

$X_{\mathrm{CO}}^{\max }=\frac{M_{v}}{M_{\mathrm{CO}}}=10 \frac{W^{2}}{D^{\max }} \frac{1-\exp \left(-\left(D_{\mathrm{arcsec}}^{\max }\right)^{2} /(1.2 \Theta)^{2}\right.}{I}$, 

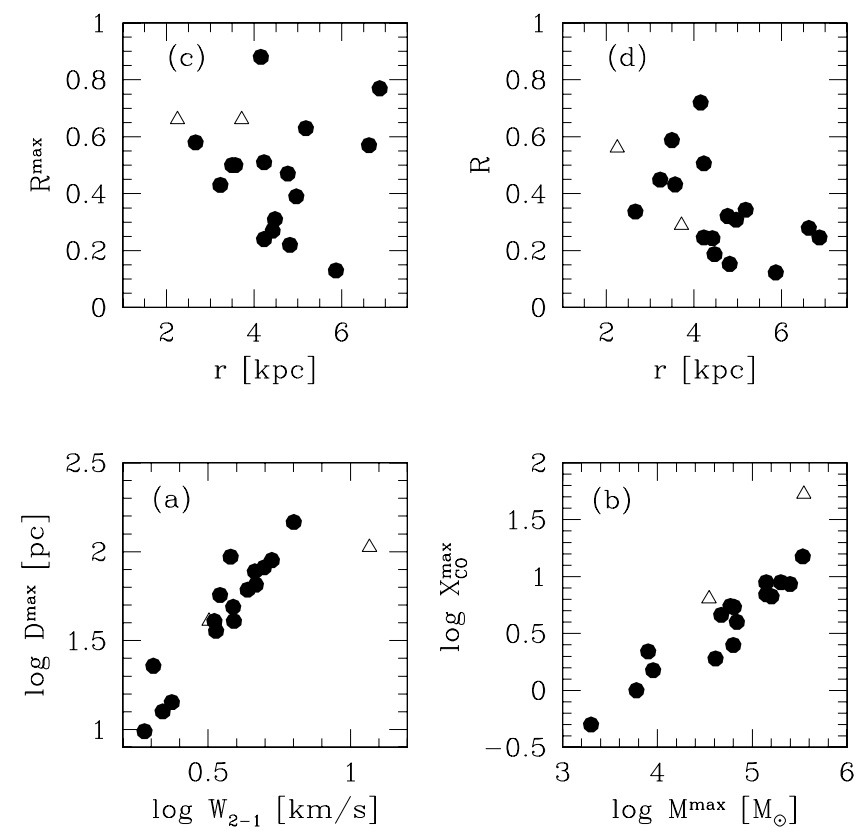

Fig. 3. Correlations using parameters derived with the second method $(\mathbf{a}), \mathbf{b}), \mathbf{c})$ ) and with the third method d)

where the values of $I, \Theta, W$ refer to the line that was used (smaller linewidth). The correlation between cloud mass and the CO-to- $\mathrm{H}_{2}$ conversion factor is shown in Fig. $3\left(r_{\mathrm{P}}=0.95\right)$. From the maximum size we can infer the maximum intrinsic line ratio, $R^{\max }$, given in Table 5 for isolated sources, at the beam center.

With this method the cloud sizes and masses are less dependent on the assumption of the cloud being centered with respect to the millimeter telescope beam. Unfortunately we are able only to derive upper limits to $M$ and $D$. In Sect. 3.4 we use the observed size-linewidth relation to infer the size of selfgravitating cloud cores. There are three sources with $X_{\mathrm{CO}}^{\max } \leq 1.5$. Those are sources with sizes smaller than $25 \mathrm{pc}$ and small masses $\left(<10^{4} M_{\odot}\right)$. Because M 33 has a lower metal abundance than the Milky Way, we do not expect an overabundance of CO. Hence, these sources are probably overluminous in $\mathrm{CO}$ or they are not in virial equilibrium (Maloney 1990). The molecular gas may be so warm that the $J=1-0$ luminosity is no longer proportional to the $\mathrm{H}_{2}$ column density. For a gas at $40 \mathrm{~K}$ for example the $J=1-0$ line intensity is more than twice as bright as for a gas at 20 K (e.g. Sakamoto et al. 1994). Recent high-resolution observations in the M 33 outer disk have discovered clouds with high CO luminosity (Bigiel et al. 2010) in the proximity of sources hosting massive stars. The very low values of $R$ that we recover for some sources may be indicative of an offcenter molecular cloud caused by the presence of massive stars. This likely holds for s15, where we detect two components, and for s20 where the $\mathrm{CO}$ observations were centered on the optical HII region in the absence of a $24 \mu \mathrm{m}$ counterpart.

\subsection{Radially varying $X_{C O}$}

In Table 6 we derive the cloud properties for a radially varying $X_{\mathrm{CO}}$ factor. The radial scaling used takes into account the weak $X_{\mathrm{CO}}$ metallicity dependence estimated by Bolatto et al. (2008)

$\log X_{\mathrm{CO}}=a-0.35 \times \log \frac{\mathrm{O}}{\mathrm{H}} \Longrightarrow X_{\mathrm{CO}}=2.8 \times 10^{20+0.03 r}$,

where the average metallicity gradient $\mathrm{O} / \mathrm{H}$ used is given by Magrini et al. (2010) and the galactocentric distance $r$ is
Table 6. Cloud parameters for a radially varying $X_{\mathrm{CO}}$.

\begin{tabular}{lcccccc}
\hline \hline ID & line & $R$ & $\begin{array}{c}D \\
\mathrm{pc}\end{array}$ & $\begin{array}{c}M \\
10^{4} M_{\odot}\end{array}$ & $\begin{array}{c}n_{\mathrm{H}_{2}}^{1-0} \\
\mathrm{~cm}^{-3}\end{array}$ & $\begin{array}{c}N_{\mathrm{H}_{2}} \\
10^{20} \mathrm{~cm}^{-2}\end{array}$ \\
\hline s1 & $2-1$ & 0.566 & 17 & 1.7 & 98 & 51 \\
s2 & & & & & & \\
s3 & $2-1$ & 0.289 & 1.2 & 1.6 & 270000 & 9990 \\
s4 & & & & & & \\
s5 & $1-0$ & 0.287 & 21 & 5.0 & 160 & 104 \\
s6 & $2-1$ & 0.670 & 56 & 2.0 & 3 & 5.2 \\
s7 & $1-0$ & 0.344 & 26 & 6.3 & 112 & 90 \\
s8 & $1-0$ & 0.441 & 33 & 3.5 & 98 & 100 \\
s9 & $1-0$ & 0.501 & 53 & 8.1 & 16 & 26 \\
s10 & $2-1$ & 0.749 & 29 & 4.2 & 53 & 47 \\
s11 & $1-0$ & 0.154 & 13 & 7.3 & 1058 & 424 \\
s12 & $1-0$ & 0.360 & 33 & 9.1 & 100 & 102 \\
s13 & $1-0$ & 0.340 & 43 & 6.4 & 24 & 32 \\
s14 & $1-0$ & 0.264 & 41 & 9.9 & 42 & 53 \\
s15 & $1-0$ & 0.254 & 26 & 2.5 & 43 & 35 \\
s15 & $2-1$ & 0.515 & 19 & 1.0 & 46 & 27 \\
s16 & & & & & & \\
s17 & $1-0$ & 0.191 & 19 & 7.7 & 360 & 211 \\
s18 & $1-0$ & 0.329 & 21 & 6.9 & 213 & 138 \\
s19 & $2-1$ & 0.249 & 13 & 5.1 & 713 & 286 \\
s20 & $1-0$ & 0.125 & 16 & 2.6 & 185 & 91 \\
\hline
\end{tabular}

Notes. The CO rotational line used to infer the cloud parameters is given in Col. 2, the intrinsic CO line ratio in Col. 3 and the cloud diameter in Col. 4. The cloud mass, the $\mathrm{H}_{2}$ volume and column density are shown in Cols. 5-7 respectively.

in kpc. The value of the constant $a$ is such that $X_{\mathrm{CO}}$ is $2.8 \times$ $10^{20} \mathrm{~cm}^{-2} \mathrm{~K}^{-1}$ at the center of M 33 . The metallicity dependence of the $X_{\mathrm{CO}}$ factor has not yet been firmly established. We prefer to consider a shallow radial dependence, because in M 33 the radial decrease of the average radiation field, which dissociates the $\mathrm{CO}$ molecule, may balance the already shallow radial metallicity gradient and keep $X_{\mathrm{CO}}$ almost constant.

We derive the source size by equating the virial to the molecular mass given by the CO line luminosity

$\frac{M_{\mathrm{vir}}}{M_{\mathrm{CO}}}=406 \frac{W^{2}}{41 X_{\mathrm{CO}} I} \frac{1-\exp \left(-D_{\mathrm{arcsec}}^{2} /(1.2 \Theta)^{2}\right.}{D_{\operatorname{arcsec}}}=1$.

From the size we infer the volume density. We then use the source size and the observed $\mathrm{CO}$ line ratio to infer the intrinsic line ratio. We take the $\mathrm{CO}$ line with the largest linewidth because it gives the highest gas density (see Eq. (6) $)^{2}$.

For isolated clouds, that are centered in the beam, the intrinsic line ratio $R$ expected for that size is also shown in Fig. 3 and decreases moving radially outward.

Limits to the validity of this method come from the fact that the weak metallicity dependence used here for $X_{\mathrm{CO}}$ is based on $\mathrm{CO}$ interferometric measurements, which may underestimate cloud sizes and hence invalidate the used scaling law (Israel \& Baas 2001). The metallicity in M 33 however has a scatter at each radius that is larger than the overall observed radial gradient: a stronger metallicity dependence can be considered only if the metallicity at each source location is known. This method is based on the assumption that clouds are in virial equilibrium. In our galaxy the data suggest that clouds with masses below

2 We use this choice with caution because one may be detecting more than one cloud in the source proximity, especially as it is often the $J=1-0$ line, observed with the largest beam, which has the largest linewidth. 
$10^{4} M_{\odot}$ are strongly influenced by nongravitational forces and virial equilibrium overestimates their masses (Maloney 1990). With only the CO observations presented here it is hard to establish if the virial equilibrium assumption is correct for clouds in our sample. If not, the clouds could be pressure-confined (as considered in Sect. 3.2) or related to short-lived events (e.g. interstellar shocks).

\subsection{Comparison with spatially resolved molecular clouds}

Recent papers (e.g. Bolatto et al. 2008; Roman-Duval et al. 2010) summarize the relevant physical properties of the Milky Way and extragalactic molecular clouds. Masses of clouds inside the solar radius mostly range between $10^{2}$ and $5 \times 10^{5} M_{\odot}$ and their sizes are below $25 \mathrm{pc}$. The median value of the average $\mathrm{H}_{2}$ volume density distribution (see Table 1 of Roman-Duval et al. 2010 , ) is $230 \mathrm{~cm}^{-3}$ with half of the clouds having densities between 100 and $400 \mathrm{~cm}^{-3}$ and with small mass clouds having the highest densities. The BIMA all-disk survey of M33 has identified GMCs with a completeness limit of $10^{5} M_{\odot}$ (Engargiola et al. 2003; Rosolowsky et al. 2007). Hence, we expect our sources to be associated to smaller mass clouds, below the completeness limit of the BIMA survey. M 33 is a low-luminosity galaxy with a lower gas and stellar surface density than the Milky Way and flocculent spiral arms. The mass spectrum of molecular clouds in M33 is steeper than that of the Milky Way (Blitz \& Rosolowsky 2005), and the average cloud properties are more similar to those of the clouds in the outer Galaxy. Molecular clouds indeed respond to large scale environmental variations. In the outer Galaxy molecular clouds have smaller masses than in the inner regions, and it is still a debated question whether clouds of small mass are pressure-bounded, or in virial equilibrium (Heyer et al. 2001; Blitz \& Rosolowsky 2005; Heyer et al. 2009).

For a quantitative comparison between the cloud properties in our sample, which we inferred using the three models presented in this section, and the resolved molecular clouds, we focus on the observed size-linewidth relation:

$W=1.03 \times D^{0.5}$,

where $W$ is the $F W H M$ of the CO line in $\mathrm{km} \mathrm{s}^{-1}$ and $D$ is the cloud diameter in parsec. The exponent of the relation was originally derived by Solomon et al. (1987), while the coefficient 1.03 comes from the best fit to resolved clouds in the Milky Way and in M 33 (Rosolowsky et al. 2003; Blitz \& Rosolowsky 2005). We use $30 \%$ larger cloud radii for M 33 data to overcome the difference in the size measurement technique with the Milky Way data pointed out by Rosolowsky et al. (2003). The above relation fits the data over two orders of magnitude in radius, from 1 to $100 \mathrm{pc}$, and we show it by the continuous line in the three panels of Fig. 4. In the same figure we plot the data for M33 GMCs (open symbols) (Wilson \& Scoville 1990; Rosolowsky et al. 2003) and for clouds with respect to the HII regions in our survey (filled circles). Cloud sizes derived using a uniform intrinsic line ratio $R=0.5$ (bottom panel) are too large to satisfy the observed size-linewidth relation. Furthermore, cloud masses are too large to be compatible with previous surveys, and the median volume density is lower by more than a factor 10 than the value in the Milky Way. Considering the outer envelope of the clouds in hydrostatic equilibrium with the ISM, we estimate higher densities. However, we cannot derive the parameters of the cloud with only this assumption, only their limiting values. To overcome this limitation, we assume that the size-linewidth

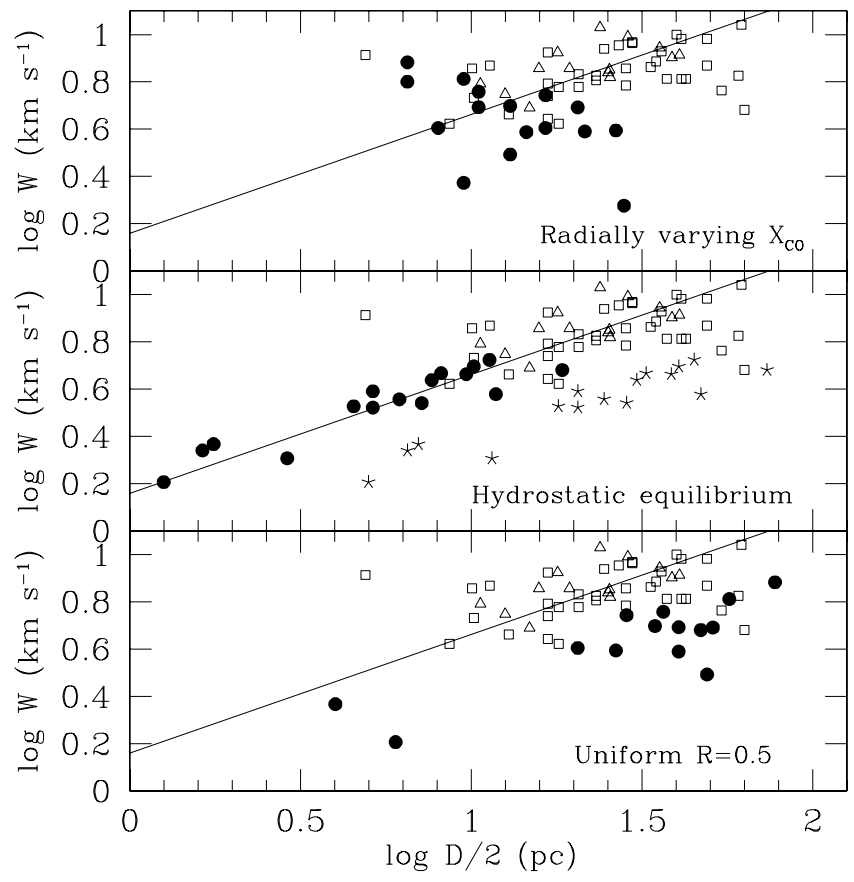

Fig. 4. Fit to the size-linewidth relation for the Milky Way and M 33 spatially resolved molecular clouds (solid line). The open symbols are for GMCs observed in M 33 by Rosolowsky et al. (2003) (open squares) and by Wilson \& Scoville (1990) (open triangles). The filled circles are molecular clouds associated to our HII regions. Sizes were computed according to the uniform intrinsic line ratio in the bottom panel and to the radially varying $X_{\mathrm{CO}}$ model in the top panel. In the middle panel asterisks indicate the maximum cloud size for hydrostatic equilibrium models and filled circles the core sizes, which are 0.25 of the maximum size. This scaling minimizes the scatter around the size-linewidth relation. The linewidths used are the CO $J=1-0$ linewidth in the bottom panel and are given in Tables 5 and 6 for the other two panels.

relation observed in the Milky Way and in the GMCs of M 33 holds for gravitationally bound cloud cores, and that there is an average scaling factor of the core size with respect to the maximum cloud size $D^{\text {max }}$ (given in Table 5 and plotted with asterisk symbols in the middle panel). By minimizing the dispersion around the plotted size-linewidth relation, we derive core sizes that are a factor 4 smaller than $D^{\max }$. These are shown in the middle panel of Fig. 4 by filled circles. In the top panel of the figure we plot cloud sizes with respect to the radially varying $X_{\mathrm{CO}}$ model (Table 6). We can see that despite the large scatter the average cloud size is smaller than that of GMCs and not much different from the size-linewidth relation predictions.

\section{IRAC colors and the search for embedded sources}

In the previous section we saw that we were able to detect $\mathrm{CO}$ in most star-forming regions and in the proximity of some evolved stars, even though $\mathrm{CO}$ lines are generally weaker in the latter case. We will now examine whether newly born clusters can be distinguished from evolved stars with IRAC colorcolor diagrams. Previous studies on closer objects (like the LMC and SMC galaxies) used IRAC color-color diagrams to separate Young Stellar Objects (hereafter YSOs) from stars or from evolved AGBs, and AGBs from HII regions (Simon et al. 2007; Cohen et al. 2007; Marengo et al. 2008; Buchanan et al. 2009; Gruendl \& Chu 2009). Models indicate that star formation regions at different stages of the protostar to star evolutionary 


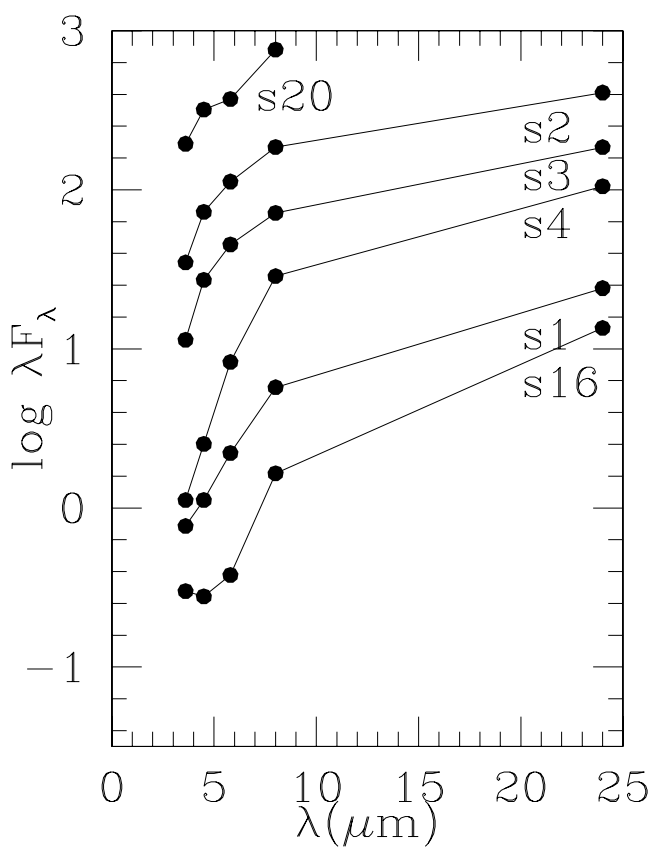

Fig. 5. IRAC SEDs: sources with a small flux increase between 4.5 and at $8 \mu \mathrm{m}$. The plot shows the sources from bottom to top scaling the fluxes so that the source at the bottom is the one with the steepest gradient between the 8 and the $24 \mu \mathrm{m}$ flux and the source at the top is the one with the shallowest gradient. Effective flux values can be recovered from Table 1. All sources except s16 show a monotonic increase of the mid-IR flux between 3.6 and $24 \mu \mathrm{m}$. The $24 \mu \mathrm{m}$ flux for s 20 is below threshold.

sequence occupy different areas of the color-color diagrams, even though there is overlapping and no well defined separation between the various areas (Robitaille et al. 2006). Similarly Gruendl \& Chu (2009) have shown that there is no simple diagnostic in color-color or color-magnitude diagrams that can be used to uniquely separate YSOs from AGB stars. Evolved stars of different types (carbon-rich versus oxygen-rich for example) lie in separate regions of the IRAC color-color diagram, but HII regions are found to lie everywhere (Buchanan et al. 2009). For AGB stars the [3.6]-[8.0] and [3.5]-[4.5] colors are used to separate carbon stars from stars with silicate envelopes owing to the presence of silicate and $\mathrm{H}_{2} \mathrm{O}$ features in the spectra. These feature affect the [3.6]-[8.0] color excess that seems to correlate with the mass-loss rate of individual stars and the optical thickness of their envelope (Marengo et al. 2008; Groenewegen 2006).

We display the mid-IR SEDs between 3.6 and $24 \mu \mathrm{m}$ in Figs. 5 and 6 . In Fig. 5 we select all sources where $\lambda F_{\lambda}$ varies by less than one order of magnitude between 5.8 and $8 \mu \mathrm{m}$, while sources with larger differences are plotted in Fig. 6. All sources in Fig. 6 have a dip at $4.5 \mu \mathrm{m}$ (see also Verley et al. 2007) except $\mathrm{s} 17$, which is the source with the lowest $\mathrm{H} \alpha$ flux. Sources are ordered according to the SED slope between 8 and $24 \mu \mathrm{m}$ with s5 being the source with the steepest gradient and s14 the source with the flatter one. For all sources in Fig. 6 we detect $\mathrm{CO}$ emission. Sources in Fig. 5 instead have either weak or no CO emission and no dip at $4.5 \mu \mathrm{m}$. We identified s1, s2, s3, and s20 as regions containing evolved variable stars. It has been shown that the MIR SED of YSOs and HII regions have steeper slopes than evolved stars (Buchanan et al. 2009; Gruendl \& Chu 2009), which agrees with our finding. This is because the SED of star-forming regions in the mid-infrared peaks at wavelengths

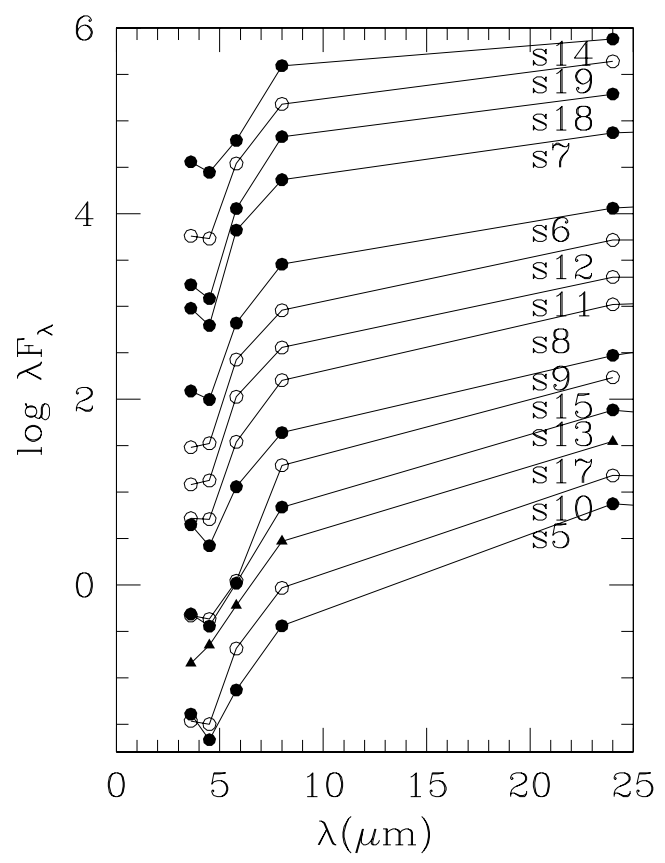

Fig. 6. IRAC SEDs: sources with a large flux growth between 4.5 and at $8 \mu \mathrm{m}$. The plot shows the sources from bottom to top scaling the fluxes so that the source at the bottom is the one with the steepest slope between the 8 and the $24 \mu \mathrm{m}$ flux, the source at the top is the one with the shallowest gradient. Effective flux values can be recovered from Table 1 . Open dots are used for sources that do not show a noticeable decrease of the flux between 3.6 and $4.5 \mu \mathrm{m}$.

longer than $24 \mu \mathrm{m}$ (e.g. Lawton et al. 2010, ). The CO emission associated with sources in Fig. 5, is weak or below our detection limit. The McQuinn et al. (2007) catalog does not extend as far as s4, which may be a variable associated with M33 or with our Galaxy. The only HII region where no $\mathrm{CO}$ has been detected is s16, likely an evolved HII region. Evolved stars and evolved HII regions show a shallow slope in the IRAC bands. The dip at $4.5 \mu \mathrm{m}$ is present in the SEDs of HII regions even though some star-forming site (s17 for example) may not have it. Some YSO SEDs show that the dip is common, but not always present (Gruendl \& Chu 2009; Simon et al. 2007). Results from ISO spectroscopy around massive protostars have shown that $\mathrm{CO}$ and $\mathrm{CO}_{2}$ absorption bands are present in the $4.5 \mu \mathrm{m}$ band (van Dishoeck 2004). These features can explain that the dip is mostly present in the SED of star-forming regions. The $\mathrm{CO}_{2}$ absorption line and a time-dependent $\mathrm{CO}$ absorption feature can also be present in the spectra of AGB stars (Marengo et al. 2010; Justtanont et al. 2004), but their strengths are not sufficient to notably affect the flux in our sample.

Figure 7 shows all long period variables cataloged by McQuinn et al. (2007) that were detected at 3.6, 4.5 and $8.0 \mu \mathrm{m}$ in the [3.6]-[8.0]/[3.6]-[4.5] and [4.5]-[8.0]/[3.6]-[4.5] colorcolor diagrams. In the same figure we plot sources from the catalog of Verley et al. (2007) with $\mathrm{H} \alpha$ emission, which are likely to be young star-forming regions, and do not have any long-term variable coincident with the extent of the $24 \mu \mathrm{m}$ emission. Obviously the variable stars occupy a well defined area and the HII regions have a distribution that is centered on a different region of the color-color diagram, with some overlap.

The two lines in Fig. 7 have slopes 1 and 0.5 respectively and separate the region populated by evolved stars. Only about $3 \%$ of the evolved stars lie to the right of the lines (about $10 \%$ of the IR selected HII regions lie to their left). The sources considered 


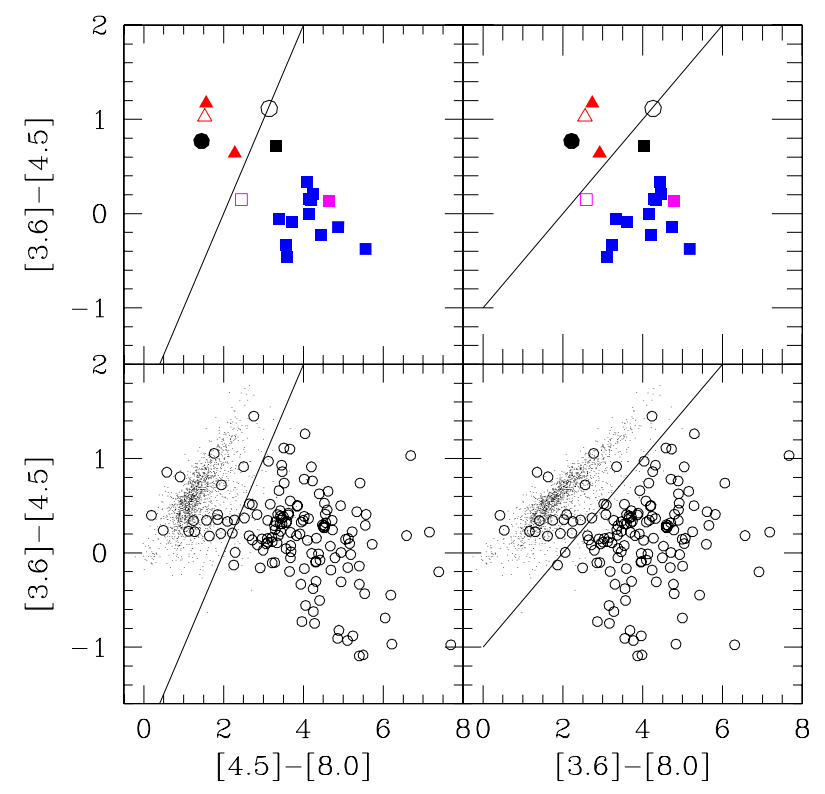

Fig. 7. Bottom panels display in the IRAC color-color diagrams the variable stars detected by McQuinn et al. (2007) (small dot symbols) and young HII regions (the open circles; these are sources in the catalog of Verley et al. (2007) with $\mathrm{H} \alpha$ counterpart and not classified as variables). Only $3 \%$ of the variable stars lie to the right of the continuous line. In the upper panels we show the same IRAC colors, but for our source sample. Open symbols are sources where no $\mathrm{CO}$ was detected, filled symbols are sources with $\mathrm{CO}$ detection. Triangles are for variable stars. The open circle is for source 4 , with uncertain classification. Squares are for sources from 5 to 19, i.e. HII regions with $24 \mu \mathrm{m}$ counterparts. The filled dot is for s20, a variable star coincident with an HII region without $24 \mu \mathrm{m}$ emission.

here well represent the two distributions. Our HII regions with a detectable $\mathrm{CO}, 24 \mu \mathrm{m}$ and $\mathrm{H} \alpha$ emission lie in a well defined region and not in the upper left corner where most of the evolved stars lie. Close to the dividing line we find s17, an embedded HII region candidate without $\mathrm{H} \alpha$ and very low UV fluxes, and s16, the HII region devoid of $\mathrm{CO}$. We cannot exclude that s16, s17 host an evolved star that did not meet the used classification schemes (they are both within a few parsec of a non-point source variable according to Hartman et al. 2006). The probability for an AGB star to encounter a star-forming region is low in the solar neighborhood (Kastner \& Myers 1994), but it can be considerable along spiral arms and filaments. The colors of s20, an evolved HII region with no $24 \mu \mathrm{m}$ counterpart that is associated to an evolved variable star, has IRAC colors similar to variable stars.

In Figure 8 we display other variables and colors, even outside the IRAC range. In each panel the young HII regions where dust and CO emission were detected occupy well defined areas. For example all of them except one (s14) have the $3.6 \mu \mathrm{m}$ magnitude above -1 and have [24]-[3.6] $<-6.5$. Similarly their IRAC color [5.8]-[8.0] is $>1.6$. There is also a nice correlation between the [4.5]-[5.8] color and the [3.6]-[8.0] color with the young HII regions where dust and $\mathrm{CO}$ emission were detected at $[4.5]-[5.8]>1$. Triangles in the upper panels of Fig. 9 indicate the colors of $24 \mu \mathrm{m}$ sources in the catalog of Verley et al. (2007) with no $\mathrm{H} \alpha$ counterpart that "hosts" a variable star, i.e. at least one classified variable is located within the $24 \mu \mathrm{m}$ emission boundary. Only 25 out of 125 sources in the catalog of Verley et al. (2007) with no $\mathrm{H} \alpha$ counterpart and detected in IRAC bands were classified as variable stars. Most of these lie to the left of the lines drawn in Fig. 9. Variable sources that lie
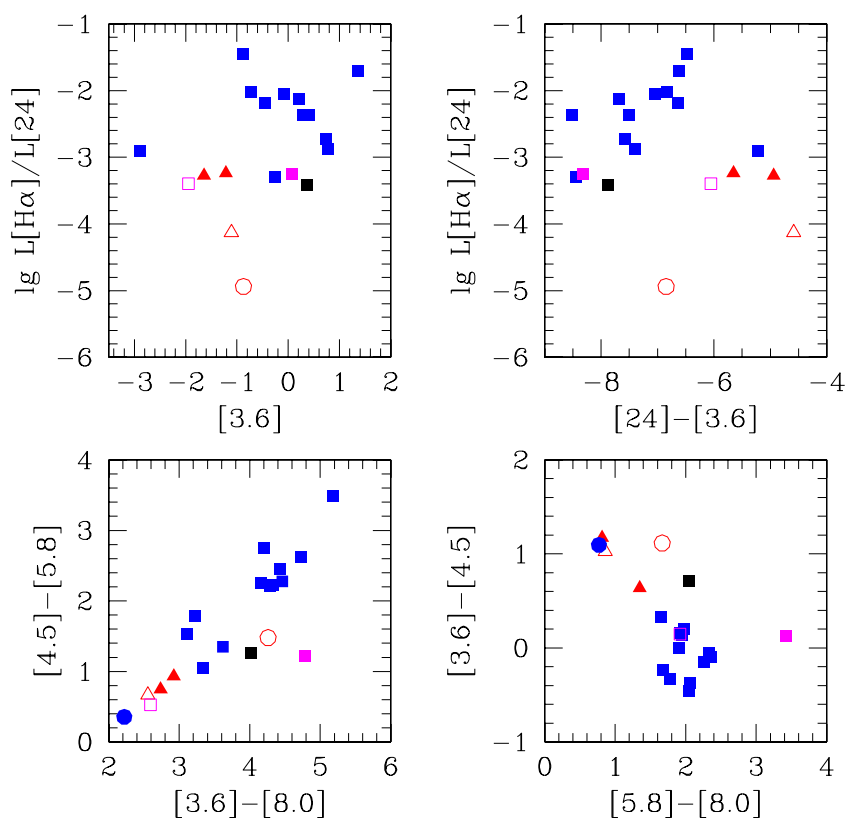

Fig. 8. Other colours, but same symbols as in the previous figure. The source with the highest flux at $3.6 \mu \mathrm{m}$ is $\mathrm{s} 14$.

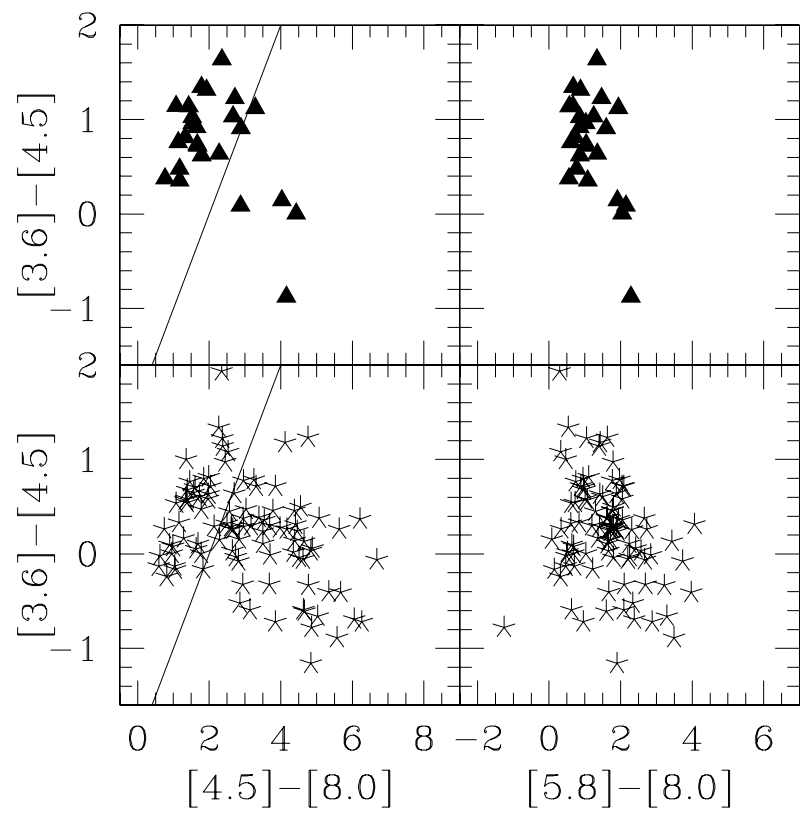

Fig. 9. Star symbols in the lower panels are for $24 \mu \mathrm{m}$ sources with no $\mathrm{H} \alpha$ counterpart that were not identified by McQuinn et al. (2007) as variable stars. Filled triangles in the upper panels are sources in Verley et al. (2007) catalog that host variable stars according to McQuinn et al. (2007).

to the right of the line may contain both an evolved star and an embedded HII region. In the bottom panels we show sources with no $\mathrm{H} \alpha$ counterpart according to the catalog of Verley et al. (2007), which are not associated to known variable stars. The number of these sources to the left of the line exceed by far the percentage expected if these sources have similar colors to the visible HII regions. Either embedded stars occupy a wider area of the IRAC color-color diagram than HII regions, or there are unidentified AGBs, variables in crowded regions that escaped the classification by McQuinn et al. (2007). The majority of sources to the right of the line are good candidates for being young star-forming regions. Only five of these sources are associated with known GMCs. The associated molecular clouds 


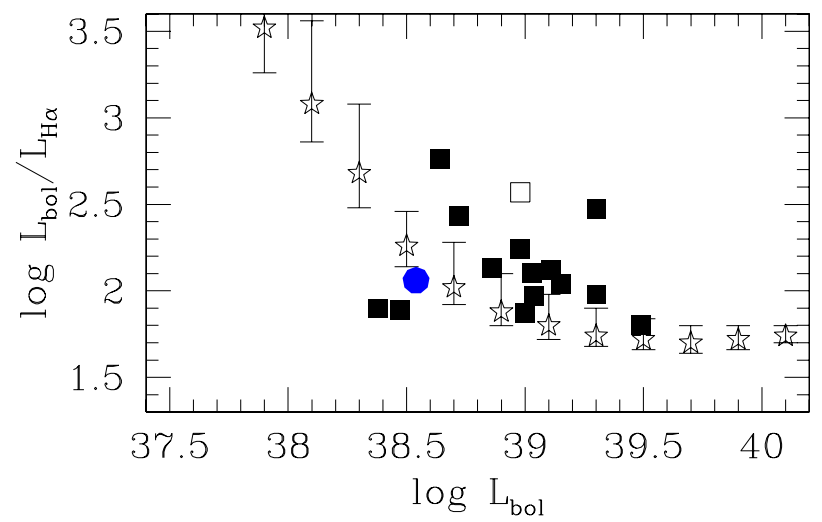

Fig. 10. Sources on the cluster birthline. Star symbols indicate the birthline with respect to a stochastically sampled universal IMF with their $1-\sigma$ dispersion. Open symbols are for sources where no $\mathrm{CO}$ was detected, filled symbols are sources with a CO detection. The filled dot is for s20, a source with no IR emission.

may be of smaller mass, like the ones detected in the CO survey presented here.

\section{The cluster birthline test}

To verify the young age of our sources, we check if they lie close to the cluster birthline, which is the line in the $L_{\mathrm{bol}}-L_{\mathrm{bol}} / L_{\mathrm{H} \alpha}$ plane around which young clusters lie (Corbelli et al. 2009). Older clusters should be located above the birthline because of the faster fading of the $\mathrm{H} \alpha$ luminosity compared with the bolometric luminosity. Leakage of ionizing photons or the ejection or the delayed formation of massive stars can also move the clusters above the birthline. In Fig. 10 we show with star symbols the birthline with respect to a stochastically sampled universal IMF with a Salpeter slope -2.35 at the high-mass end. Stochasticity allows a spread in the values of $L_{\mathrm{bol}} / L_{\mathrm{H} \alpha}$ for a given $L_{\mathrm{bol}}$ : for a given bolometric luminosity we can have a fully populated IMF up to a certain mass $M_{\max }$, or an IMF populated only up to $M_{*}<M_{\max }$, plus a brighter outlier. The errorbars in each bin indicate the dispersion caused by bright outliers that can form for the given $L_{\mathrm{bol}}$. The highest simulated $L_{\mathrm{bol}} / L_{\mathrm{H} \alpha}$ value is for a fully populated IMF case. We see that there are two sources $(\mathrm{s} 12, \mathrm{~s} 16)$ which are clearly above the upper 3- $\sigma$ boundary. These may be more evolved sources or leaking ionizing radiation. Because we did not find $\mathrm{CO}$ emission from s16, it is likely that it is an evolving source. The source s12 is the brightest in the IR and has a strong CO emission: thus, its position can be explained if it is in the process of forming its massive stellar population or if it is leaking ionizing photons. The strong $8 \mu \mathrm{m}$ emission places s12 in the upper right corner of the [3.6]-[8.0]-[4.5]-[5.8] color plot. All the other sources cannot be found below the birthline if the IMF assumptions are correct. Our sources are all compatible with a random sampled IMF model including the two sources, s13 and s19 close to the lower boundary, that may host outlier stars.

\section{Conclusions}

We investigated the nature of faint $24 \mu \mathrm{m}$ sources with a weak or no $\mathrm{H} \alpha$ counterpart. These could be regions with embedded clusters associated to molecular gas, or evolved stars with dusty envelopes. To distinguish between these two possibilities, we used published catalogs of variable stars in M 33 and carried out deep observations of the CO $J=1-0$ and $2-1$ line with the
IRAM 30-mt telescope to search for molecular gas associated with 20 sources. The main results are:

- Deep pointed CO observations have revealed the presence of $\mathrm{CO}$ around 17 of the 20 sources included in our sample. The weakness of the $\mathrm{CO}$ lines around most of our sources is indicative of the existence of a large population of faint $\mathrm{CO}$ clouds in M33. This is in agreement with the results of the CO survey of Gratier et al. (2010) and with the steeper molecular mass spectrum with respect to our Galaxy (Blitz \& Rosolowsky 2005).

- The estimate of cloud masses is uncertain because our pointed observations are deeper than any existing CO map. Considering clouds in virial equilibrium and a weak radial dependence of the $\mathrm{CO}-$ to- $\mathrm{H}_{2}$ conversion factor, we find molecular gas masses in the range $10^{4}-10^{5} M_{\odot}$. In this case, cloud sizes agree on average with those predicted by the sizelinewidth relation of the Milky Way and resolved GMCs in other galaxies, but have large dispersions. We also estimated limits to molecular gas masses and sizes in the case where cloud outer envelopes are in pressure equilibrium with the surrounding medium. This model reproduces the observed size-linewidth relation correctly if gravitationally bound cloud cores are a factor 4 smaller in size than the outer envelopes.

- The intrinsic CO $J=2-1$ to $J=1-0$ line ratio is generally small, $\sim 0.4$, if one makes the assumption that clouds are within a few parsecs from the beam center. The CO lines are weaker at the location of evolved stars than around starforming regions, as is the $\mathrm{H} \alpha$ and $70 \mu \mathrm{m}$ emission. Stellar clusters associated to clouds in our sample are young because they lie along the cluster birthline.

- In the IRAC color-color diagrams AGB variable stars and HII regions occupy distinct areas even though there is some overlap: most of the AGBs have [3.6]-[4.5] color in the range 0 to 1.5 and lie around a line of slope 0.5 in the [3.6]-[8.0][3.6]-[4.5] plane. Most of the $24 \mu \mathrm{m}$ sources associated to HII regions have higher values for the [4.5]-[8.0] or [3.6][8.0] color than variable stars. The IRAC colors of our 20 selected sources are consistent with these distributions. They show a linear correlation between the [4.5]-[5.8] color and the [3.6]-[8.0] color with the AGBs having the lowest values. The IRAC colors confirm the presence of AGBs in four sources of our sample, whose variability was previously detected either in infrared or optical surveys.

- Using IRAC color-color diagrams we also predict that in the sample of $24 \mu \mathrm{m}$ sources without an $\mathrm{H} \alpha$ counterpart there are still unidentified evolved stars other than young clusters. The young clusters may lack $\mathrm{H} \alpha$ emission because they are of small mass or because they are very compact, and deeply embedded. Additional sensitive $\mathrm{CO}$ observations and high-resolution far-infrared images will help to unambiguously identify the properties of the young star-forming sites in M33.

Acknowledgements. We thank the staff in Granada for their assistance during the IRAM 30-mt observations and the referee for comments to the original manuscript. The study presented in this paper was supported by the European Community Framework Programme 7, Advanced Radio Astronomy in Europe, grant agreement No.: 227290.

\section{References}

Bigiel, F., Bolatto, A. D., Leroy, A. K., et al. 2010, ApJ, 725, 1159

Blitz, L., \& Rosolowsky, E. 2005, in Astrophysics and Space Science Library, Vol. 327, The Initial Mass Function 50 Years Later, ed. E. Corbelli, F. Palla, \& H. Zinnecker, 287 
Bolatto, A. D., Leroy, A. K., Rosolowsky, E., Walter, F., \& Blitz, L. 2008, ApJ, 686, 948

Buchanan, C. L., Kastner, J. H., Hrivnak, B. J., \& Sahai, R. 2009, AJ, 138, 1597

Buckalew, B. A., Kobulnicky, H. A., Darnel, J. M., et al. 2006, ApJS, 162, 329

Cohen, M., Green, A. J., Meade, M. R., et al. 2007, MNRAS, 374, 979

Corbelli, E. 2003, MNRAS, 342, 199

Corbelli, E., Verley, S., Elmegreen, B. G., \& Giovanardi, C. 2009, A\&A, 495, 479

Corbelli, E., Giovanardi, C., \& Grossi, M. 2010 [arXiv: 1011. 1097]

Crosthwaite, L. P., \& Turner, J. L. 2007, AJ, 134, 1827

Engargiola, G., Plambeck, R. L., Rosolowsky, E., \& Blitz, L. 2003, ApJS, 149, 343

Fazio, G. G., Hora, J. L., Allen, L. E., et al. 2004, ApJS, 154, 10

Freedman, W. L., Wilson, C. D., \& Madore, B. F. 1991, ApJ, 372, 455

Gardan, E., Braine, J., Schuster, K. F., Brouillet, N., \& Sievers, A. 2007, A\&A, 473, 91

Gil de Paz, A., Boissier, S., Madore, B. F., et al. 2007, ApJS, 173, 185

Glover, S. C. O., \& Mac Low, M. 2011, MNRAS, 110

Gratier, P., Braine, J., Rodriguez-Fernandez, N. J., et al. 2010, A\&A, 522, A3

Greenawalt, B. E. 1998, PhD Thesis, AA (New Mexico State University)

Groenewegen, M. A. T. 2006, A\&A, 448, 181

Groenewegen, M. A. T., Wood, P. R., Sloan, G. C., et al. 2007, MNRAS, 376, 313

Grossi, M., Corbelli, E., Giovanardi, C., \& Magrini, L. 2010, A\&A, 521, A41

Gruendl, R. A., \& Chu, Y. 2009, ApJS, 184, 172

Hartman, J. D., Bersier, D., Stanek, K. Z., et al. 2006, MNRAS, 371, 1405

Heyer, M. H., Carpenter, J. M., \& Snell, R. L. 2001, ApJ, 551, 852

Heyer, M. H., Corbelli, E., Schneider, S. E., \& Young, J. S. 2004, ApJ, 602, 723

Heyer, M., Krawczyk, C., Duval, J., \& Jackson, J. M. 2009, ApJ, 699, 1092

Hoopes, C. G., \& Walterbos, R. A. M. 2000, ApJ, 541, 597

Israel, F. P., \& Baas, F. 2001, A\&A, 371, 433

Justtanont, K., de Jong, T., Tielens, A. G. G. M., Feuchtgruber, H., \& Waters,

L. B. F. M. 2004, A\&A, 417, 625

Kastner, J. H., \& Myers, P. C. 1994, ApJ, 421, 605

Lawton, B., Gordon, K. D., Babler, B., et al. 2010, ApJ, 716, 453
Leitherer, C., Schaerer, D., Goldader, J. D., et al. 1999, ApJS, 123, 3

Magrini, L., Stanghellini, L., Corbelli, E., Galli, D., \& Villaver, E. 2010, A\&A, 512, A63

Makovoz, D., \& Khan, I. 2005, in Astronomical Data Analysis Software and Systems XIV, ed. P. Shopbell, M. Britton, \& R. Ebert, ASP Conf. Ser., 347, 81

Maloney, P. 1990, ApJ, 348, L9

Maloney, P., \& Black, J. H. 1988, ApJ, 325, 389

Marengo, M., Reiter, M., \& Fazio, G. G. 2008, in Evolution and Nucleosynthesis in AGB Stars, ed. R. Guandalini, S. Palmerini, \& M. Busso, AIP Conf. Ser., 1001, 331

Marengo, M., Evans, N. R., Barmby, P., et al. 2010, ApJ, 709, 120

Martin, D. C., Fanson, J., Schiminovich, D., et al. 2005, ApJ, 619, L1

McQuinn, K. B. W., Woodward, C. E., Willner, S. P., et al. 2007, ApJ, 664, 850

Oka, T., Hasegawa, T., Hayashi, M., Handa, T., \& Sakamoto, S. 1998, ApJ, 493, 730

Rieke, G. H., Young, E. T., Engelbracht, C. W., et al. 2004, ApJS, 154, 25

Robitaille, T. P., Whitney, B. A., Indebetouw, R., Wood, K., \& Denzmore, P. 2006, ApJS, 167, 256

Roman-Duval, J., Jackson, J. M., Heyer, M., Rathborne, J., \& Simon, R. 2010, ApJ, 723, 492

Rosolowsky, E., Engargiola, G., Plambeck, R., \& Blitz, L. 2003, ApJ, 599, 258

Rosolowsky, E., Keto, E., Matsushita, S., \& Willner, S. P. 2007, ApJ, 661, 830

Sakamoto, S., Hayashi, M., Hasegawa, T., Handa, T., \& Oka, T. 1994, ApJ, 425, 641

Simon, J. D., Bolatto, A. D., Whitney, B. A., et al. 2007, ApJ, 669, 327

Solomon, P. M., Rivolo, A. R., Barrett, J., \& Yahil, A. 1987, ApJ, 319, 730

Sorai, K., Hasegawa, T., Booth, R. S., et al. 2001, ApJ, 551, 794

Spitzer, L. 1978, Physical processes in the interstellar medium, ed. L. Spitzer

Thilker, D. A., Hoopes, C. G., Bianchi, L., et al. 2005, ApJ, 619, L67

van Dishoeck, E. F. 2004, ARA\&A, 42, 119

Verley, S., Hunt, L. K., Corbelli, E., \& Giovanardi, C. 2007, A\&A, 476, 1161

Verley, S., Corbelli, E., Giovanardi, C., \& Hunt, L. K. 2009, A\&A, 493, 453

Verley, S., Corbelli, E., Giovanardi, C., \& Hunt, L. K. 2010, A\&A, 510, A64

Werner, M. W., Roellig, T. L., Low, F. J., et al. 2004, ApJS, 154, 1

Wilson, C. D., \& Scoville, N. 1990, ApJ, 363, 435 